\title{
Alterações fisiológicas e biomecânicas em indivíduos praticando exercícios de hidroginástica dentro e fora d'água
}

\author{
KRUEL, Luiz Fernando Martins ${ }^{1}$, MORAES, Eliane Zenir Correa de ${ }^{2}$, \\ ÁVILA, Aluisio Otávio de Vargas ${ }^{3}$ e SAMPEDRO, Renan Maximiliano Fernandes ${ }^{4}$
}

\section{Resumo}

O objetivo deste trabalho foi comparar alterações fisiológicas e biomecânicas durante exercícios de hidroginástica praticados fora d'água (FD) e nas profundidades de água de cicatriz umbilical (PCU) e de ombro (PO). A amostra foi composta por 23 indivíduos do sexo feminino, com idade média de $54 \pm 11,16$ anos, praticantes de hidroginástica, participantes do programa de extensão universitária da Escola de Educação Física da UFRGS. A amostra inicialmente foi subdividida em cinco grupos (um grupo para cada exercício de hidroginástica, sorteados entre os exercícios mais utilizados pelos professores de hidroginástica do Brasil). Os exercícios sorteados foram o Garça, Lagosta, Jacaré I e II e o Pelicano. A formação dos grupos experimentais foi feita aleatoriamente. Cada exercício foi executado por 5 minutos e o tempo de recuperação entre uma execução e outra foi determinado de forma individual. As variáveis analisadas foram: frequiência cardíaca (FC), concentração de lactato sangüíneo (Lac), consumo de oxigênio $\left(\mathrm{VO}_{2}\right)$, força de reação vertical $(\mathrm{Fz})$ e o impulso (Imp). Diferentes tratamentos estatísticos foram utilizados para comparar as classes de variáveis classificatórias, para a localização das diferenças, para verificar a correlação entre as variáveis fisiológicas e biomecânicas e para determinar o grau de influência de uma variável sobre outra, usando-se o pacote estatístico SPSS for Windows versão 8.0. Não foram encontradas diferenças estatisticamente significantes nas variáveis analisadas entre os cinco exerčcios. realizados, com exceção do $\mathrm{VO}_{2}$ fora d'água entre o exercício Lagosta e o Pelicano. O comportamento dę ţ̣das as variáveis estudadas foi semelhante, apresentando maiores valores quando os indivíduos realizavam o exercício FD, e diminuindo a medida que aumentava a profundidade de imersão. $\mathrm{VO}_{2}$ e FC não mostraram fififerenças estatisticamente significantes entre FD e PCU, mas mostraram diferenças entre a PO e os outros dois tratamentos (FD e PCU), o mesmo acontecendo para Lac, Imp e Fz. Nos exercícios realizados FD as variáveis fisiológicas tem um maior poder explicativo do fenômeno analisado, enquanto que na PCU e PO são as variáveis biomecânicas que tem um maior poder explicativo dos fenômenos analisados.

Palavras-Chaves: hidroginástica, freqüência cardíaca, consumo de oxigênio.

\footnotetext{
1 Prof. Dr. Universidade Federal do Rio Grande do Sul.

${ }^{2}$ Prof. mestranda do Programa de Pós-Graduação em Ciência do Movimento Humano do CEFD, Universidade Federal de Santa Maria.

${ }^{3}$ Prof. Dr. Universidade do Estado de Santa Catarina.

${ }^{4}$ Prof. Dr. da Universidade de Cruz Alta.
}

Revista Kinesis, Santa Maria, $N^{\circ}$ Especial, p. 104-154, nov. 2001 


\section{Introdução}

A água é um meio ambiente diferente do ar em vários aspectos. No entanto, o homem, que está adaptado ao ar, insiste em entrar neste ambiente estranho para o trabalho, recreação, higiene e terapia. Estas atividades freqüentemente envolvem exercício, e todo o movimento nos desportos é influenciado pelo meio em que ocorre a atividade (Kruel, 1994).

Na concepção de Avellini et al. (1983) pode-se então esperar que o exercício físico aquático produza reações fisiológicas diferentes daqueles ao ar livre devido, tanto ao efeito hidrostático da água nos sistemas cardio-respiratórios, como à sua capacidade de intensificar a perda de calor comparada ao ar.

A imersão no meio líquido expõe o corpo humano a uma nova pressão hidrostática (Agostini et al., 1966; Rennie et al., 1971; Denison et al., 1972; Arborelius et al., 1972a; Arborelius et al., 1972b; Etcht et al., 1974; Lange et al., 1974; Gauer \& Henry, 1976; Heigenhauser et al., 1977; Blomqvist et al., 1980; Avellini et al., 1983; Blonqvist \& Stone, 1983, Lin, 1984; Sheldahl et al., 1984 e Greenleaf et al., 1988) a outra viscosidade do meio (Denison et al., 1972), a novas condições térmicas (Keatinge \& Evans, 1961; Kawakami et al., 1967; Costill et al., 1967; Rennie et al., 1971; Denison et al., 1972; Arborelius et al., 1972a; Hölmer \& Bergh, 1974; Folinsbee, 1974, Echt et al., 1974; Mcardle, 1976; Mcmurray \& Horwath, 1979, Avellini et al., 1983; Sheldahl, 1985 e Choukroun \& Varene, 1990), e a estímulos reflexos (Denison et al., 1972; Arborelius et al., 1972a e Gauer \& Henry, 1976), que poderiam alterar as respostas cardio-circulatórias durante o exercício e em repouso. Os efeitos destas trocas, de acordo com Denison et al. (1972), podem variar com alterações na postura, na intensidade de trabalho, tipo de movimento de braços; temperatura da água e deveriam ser mais evidentes em exercícios máximos em água fria.

Para Sheldahl (1985), a modalidade mais convencional de exercícios aquáticos é a natação. Suas vantagens incluem um mínimo estresse nas articulações que suportam peso, redução do estresse relativo ao calor em água fresca e a possibilidade de exercitar-se numa capacidade aeróbica ideal, desde que o indivíduo tenha relativo domínio da técnica dos estilos de nado. A maior desvantagem da natação é que muitos indivíduos não tem habilidades adequadas para exercitar-se num nível aeróbico ideal.

Conforme Cassady \& Nielsen (1992) uma outra forma de exercícios aquáticos - a calistenia na água (hidroginástica) - pode ser uma alternativa viável aos exercícios em terra para indivíduos com artrites, com dores nas costas e para vários tipos de disfunçõẻs ortopédicas que tenham dificuldades com os componentes de peso-apoio dos exercícios térestres. Estes exercícios na água estão crescendo em popularidade. Tanto os adeptos tradicionais de atividades físicas, bem como as pessoas que estão iniciando uma atividade (indivíduos com sobrepeso, gestantes, sedentários e idosos) podem participar desta modalidade de exercício.

Na hidroginástica vários tipos de caminhadas, corridas, saltos e chutes são realizados na água, em diferentes profundidades. A maior vantagem destes tipos de exercícios segundo Evans et al. (1978), Sheldahl (1985), Whitley \& Schoene (1987), Cassady \& Nielsen (1992), Wilder \& Brennan (1993), Wilder et al. (1993) e Wilber et al. (1996) é a menor incidência de estresse nas articulações comparada aos mesmos exercícios realizados na terra, principalmente, para indivíduos com problemas nas articulações dos membros inferiores e de peso. E, ainda, exercícios que talvez não atinjam um mínimo nível aeróbico na terra (ex.: caminhada lenta) talvez resultem 
em um treinamento adequado na água, devido ao aumento da resistência da mesma durante o movimento.

De acordo com Cassady \& Nielsen (1992) apesar dos benefícios dos exercícios aquáticos, poucas pesquisas tem sido conduzidas para comparar as respostas fisiológicas de exercícios realizados em terra ou na água.

Para Bullard \& Rapp (1970) e Evans et al. (1978) há duas características de imersão na água que talvez sejam especialmente benéficas num programa de exercícios. Primeiro o efeito de flutuação na imersão, pois o mesmo reduz o estresse nas articulações que suportam peso, comparado a exercícios feitos no solo. Uma vez que os efeitos de boiar e resistência da água tornam possíveis altos níveis de gasto de energia com relativamente pouco movimento e esforço das articulações dos membros inferiores, isto talvez permita um maior e mais rápido progresso em termos de intensidade, freqüência e duração dos exercícios, assim como diminui o risco de ocorrer traumatismos nas articulações que suportam peso durante o exercício. Em termos de pressão da articulação, quanto mais pesado o indivíduo, maior as forças de impacto nas articulações do pé, tornozelo e pelve. Esta pressão aumenta significativamente da caminhada para a corrida ou dança aeróbica de alto impacto e pode causar vários tipos de lesões relacionadas ao esforço nestas articulações.

Uma segunda característica da imersão na água, segundo os autores anteriormente citados, que talvez seja benéfica num programa de exercícios é o alto calor específico e a termocondutividade da água comparada com a do ar, as quais aumentam a capacidade de remover calor do corpo em água fresca. Para Sheldahl (1985), isto pode resultar num menor estresse durante o exercício, o que pode ser especialmente benéfico para os obesos, visto que há indicações que eles não cheguem a atingir alto esforço e calor comparados ao estresse associado a indivíduos de peso normal.

Com a imersão vertical do corpo na água, tanto em exercícios aquáticos, exercícios na bicicleta ergométrica, caminhadas na água ou outras situações, deve ser observado que há um gradiente hidrostático de pressão exercido na superfície do corpo. Devido a este gradiente de pressão da água, há uma transferência de sangue venoso das extremidades inferiores e do abdômen para a região torácica, resultando num aumento do volume central de sangue (Sheldahl, 1985).

No entender de Cunha et. al. (1995) a análise do movimento é um recurso importante para o estudo e a compreensão da motricidade humana. Com ela pode-se não só medir e descrever o movimento, como também compreender de que forma ele é controlado (Zatsiorsky \& Fortney, 1993).

As técnicas utilizadas na biomecânica, para Oliveira et al. (19்̧ 95 ), são elos importantes de integração com as análises do movimento na área de fisiolổia do exercício. A biomecânica para Amadio (1993) deriva-se das ciências naturais, e se ocupa das análises físicas do sistema biológico e, consequentemente de análises do movimento humano. $\mathrm{O}$ estudo do movimento resulta de investigações que são obtidas pelo uso de métodos e técnicas próprias.

Para Sacco \& Amadio (1995) a descrição quantitativa de aspectos biomecânicos do movimento humano seguramente está ligada às forças que causam o movimento observado, assim como suas repercussões no fenômeno analisado. As forças que agem no corpo humano podem ser divididas em duas categorias: as forças internas, como a força muscular por exemplo, e as forças externas que determinam todas as interações físicas entre o corpo e o meio ambiente, por exemplo, as forças de reação do solo. Portanto, o movimento humano apresenta-se Revista Kinesis, Santa Maria, $N^{o}$ Especial, p. 106-154, nov. 2001 
estruturalmente modificado de acordo com as características próprias de cada indivíduo, sua morfologia, tipo de atividade, idade e presença de determinadas patologias, entre outros fatores.

A investigação da força de reação do solo na fase de apoio dos movimentos de locomoção, bem como da distribuição de pressão dinâmica na superfície plantar, traz importantes conhecimentos sobre a forma e características da sobrecarga mecânica sobre o aparelho locomotor humano e seu comportamento para movimentos selecionados. Por isso informações referentes a estas variáveis podem ainda revelar sobre a estrutura e função do pé, o controle postural, ou ainda sobre o controle do movimento.

São reconhecidas as altas forças de impacto associadas à execução de diferentes atividades esportivas, como a dança aeróbica (Francis et al., 1988 e Michaud et al., 1993), o movimento de rebote do basquetebol (Valiant \& Cavanagh, 1985) e aterrissagens da ginástica olímpica (Ozguven \& Berne, 1988). Entretanto ao revisar a literatura constatou-se que nada tem sido investigado acerca dos níveis de sobrecarga que resultam da prática da hidroginástica.

Reconhecer variáveis físicas e fisiológicas que possam ser determinantes sobre a regulação de uma posição corporal é uma tarefa importante, pois permite dentre outros aspectos, que um observador externo possa inferir e diferenciar movimentos visualmente semelhantes, entretanto em profundidades de água e meios diferentes.

Além disto, é importante buscar os conhecimentos de outras áreas a fim de complementar a compreensão deste fenômeno. Neste caso, tanto a Fisiologia como a Aprendizagem Motora aparecem muito próximas da Biomecânica no sentido de embasar a análise do movimento.

Encontrou-se muitos estudos na literatura a respeito de alterações fisiológicas no meio líquido. A análise fisiológica, no entendimento do pesquisador, não é suficiente para compreender claramente os fenômenos investigados no meio aquático. Necessita-se, então, associar as informações fisiológicas obtidas com aquelas provenientes de outras áreas do conhecimento científico, buscando formar um campo de trabalho com ampla possibilidade de aplicação e integração. Dentre estas, a integração entre variáveis biomecânicas e fisiológicas parecem ser um bom exemplo, apesar dos trabalhos encontrados na literatura serem poucos e não conclusivos, de uma forma geral, e inexistentes quando tentou-se relacionar as alterações fisiológicas de FC, consumo de oxigênio $\left(\mathrm{VO}_{2}\right)$ e Lactato Sangüíneo com variáveis biomecânicas de força de reação do solo exercida na vertical, por exemplo, em diferentes profundidades de água, uma vez que até bem pouco tempo não existia uma plataforma de força que fosse possível ser colocada no meio líquido. A partir da tese de Roesler (1997), que desenvolveu uma platafgrma de força para aquisição de forças e momentos no três eixos ( $\mathrm{x}, \mathrm{y}$ e $\mathrm{z}$ ), isto se torna possível.

De acordo com Lopes et al. (1995), um dos problemas mais críticos na avaliação fisiológica e biomecânica de indivíduos é a aplicação dos resultados em situação real da modalidade. Se de um lado, o controle laboratorial de uma série de avaliações orgânicas favorece a confiabilidade dos resultados, de outro, muitas vezes não representa auxílio na preparação física dos indi víduos, pelo distanciamento das situações envolvidas. Assim sendo, cada vez mais trabalhos associando a fisiologia do exercício e a biomecânica têm se preocupado em desenvolver avaliações no campo de trabalho real de uma determinada modalidade. Nessas situações, o controle do esforço realizado se torna crítico na validade do teste. Complementando este raciocínio, Corsino et al. (1995), dizem que a literatura científica indica que para avaliar as respostas fisiológićas e metabólicas em indivíduos deve-se tentar reproduzir os movimentos específicos requeridos pela atividade física durante o processo de medição. Somente assim será possível obter resultados 
confiáveis das respostas fisiológicas e metabólicas na atividade de interesse.

Baseado no que foi exposto e utilizando as metodologias e recursos técnicos que foram desenvolvidos pela Universidade Federal do Rio Grande do Sul e Universidade Federal de Santa Maria através dos seus laboratórios e grupos de pesquisa e também devido a grande utilização atualmente da hidroginástica por profissionais de Educação Física pretende-se com este estudo verificar:

Quais são as alterações fisiológicas e biomecânicas em indivíduos praticando exercícios de hidroginástica fora d'água e em duas diferentes profundidades de água?

\section{Metodologia}

\section{Amostra}

A amostra foi composta por 23 indivíduos do sexo feminino, com idade entre 40 e 70 anos, praticantes de hidroginástica há pelo menos seis meses, que não eram portadores de nenhum tipo de problema físico, participantes do programa de extensão universitária em hidroginástica da Escola de Educação Física da Universidade Federal do Rio Grande do Sul. A amostra foi subdividida em cinco grupos (um grupo para cada exercício de hidroginástica escolhido).

\section{Procedimentos para seleção da amostra}

Os alunos dos programas de extensão em hidroginástica da ESEF-UFRGS foram selecionados por voluntariedade. Os mesmos foram convidados através de cartazes e comunicações verbais em sala de aula a participarem da pesquisa. Os voluntários compareceram em data e horário pré-estabelecido para a coleta de dados e para o sorteio do exercício (Figuras $1,2,3,4,5)$ que iriam realizar durante o experimento.

Figura 1

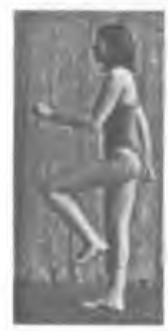

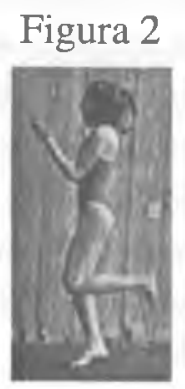

\section{Instrumentos de medida}

Figura 3

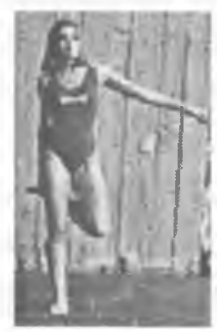

Figura 4

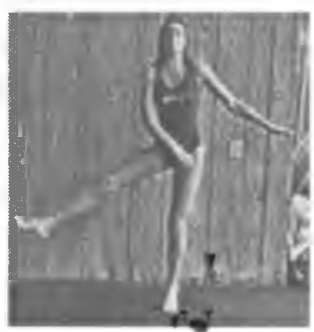

$\sigma$
Figura 5

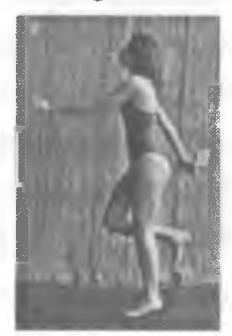

Para esta pesquisa foram utilizados os equipamentos disponíveis no Laboratório de Pesquisa do Exercício da UFRGS (LAPEX-UFRGS), no Laboratório de Pesquisa e Ensino do Movimento Humano da UFSM (LAPEM-UFSM), no Laboratório de Medições Mecânicas da UFRGS (LMM-UFRGS) e na IMBRAMED-TECNIMED.

\section{Ficha de Dados Individuais}

Para a coleta de dados utilizou-se uma ficha de dados pessoais, na qual constava: nome do indivíduo, idade, tempo de prática em hidroginástica, peso, estatura, nome do exercício a ser executado, medidas de $\mathrm{FC}, \mathrm{VO}_{2}$, LACTATO, tempo de recuperação. 


\section{Plataformas de Forca}

A obtenção de dados fisiológicos e biomecânicos fidedignos é fator preponderante para a qualidade da análise, por isso, a importância da metodologia empregada, a qual depende do uso e construção de equipamento adequado e de suas disponibilidades. Para este estudo, uma vez que não existia plataformas de força que pudessem ser imersas em meio líquido devido aos seus componentes eletro-eletrônicos, foi necessária a construção de uma plataforma de força que pudesse ser colocada dentro do meio líquido. A mesma foi desenvolvida como tema da tese de doutorado do Prof. Hélio Roessler, junto ao Laboratório de Vibrações da UFRGS.

A finalidade da plataforma de força é medir as forças de reação do solo, e os respectivos momentos dos exercícios, decompostos em 3 componentes segundo os eixos cartesianos $\mathrm{x}$, y e $\mathrm{z}$.

Neste trabalho foram utilizadas três plataformas de força, 2 subaquáticas modelo PL2 (uma para cada profundidade de água) projetadas e desenvolvidas na UFRGS, e 1 plataforma fora d'água da marca AMTI.

As plataformas PL2, tem as seguintes características:

- dimensões: 500 x $500 \mathrm{~mm}$ (de modo a permitir que uma pessoa fique em pé sobre a plataforma com folga);

- carga máxima/sensibilidade: 4000 N/2N (como a carga máxima sobre a plataforma é proporcional à sensibilidade na direção vertical, escolheu-se adotar como parâmetros de projeto a carga máxima de $4000 \mathrm{~N}$ e, consequentemente sensibilidade de aproximadamente $2 \mathrm{~N}$. Para fora d'água estes valores seriam baixos visto que em um salto uma pessoa de $80 \mathrm{~kg}$ poderia danificar uma plataforma, mas para baixo d’água dificilmente a carga máxima será atingida);

- frequiência natural: $25 \mathrm{~Hz}$;

- matéria prima: aço SAE 1010 galvanizado (porque o alumínio é corroído rapidamente na atmosfera de uma piscina);

- erro: $1 \%$.

A plataforma AMTI modelo OR6-5 tem as seguintes características:

- dimensões: 508 x 464 mm (de modo a permitir que uma pessoa fique em pé sobre a plataforma com folga);

- carga máxima/sensibilidade: $10000 \mathrm{~N} / 0,67 \mathrm{~N}$;

- matéria prima: aço;

Esta plataforma (AMTI) utiliza ainda para o sistema de condicionamento de sinal, aquisição e pós processamento: o sistema PEAK5 versão 5.3 mais um computador 486 de 50 $\mathrm{MHz}$ com 16 Mbytes de memória RAM.

\section{Balança}

O instrumento utilizado para determinação da massa corporal, foi uma balança de alavanca da marca FILIZOLA, com resolução de 100 gramas.

\section{Estadiômetro}

Para medir a estatura foi utilizado um estadiômetro de madeira. O estadiômetro é constituído de uma escala métrica, na qual desliza um cursor que mede a estatura do indivíduo na posição em pé. Esta escala é fixa a uma base apoiada ao solo, com resolução de $1 \mathrm{~mm}$. 


\section{Sensor de Batimentos Cardíacos}

Foi utilizado um sensor de batimento cardíaco, marca POLAR, modelo VANTAGE XL, que tem como características:

- indicar simultaneamente a FC, o tempo total de exercício e a hora atual;

- poder armazenar na memória oito diferentes arquivos com trinta e três horas de monitorização;

- gravar e armazenar histórico detalhado para posterior avaliação em computador;

- ser a prova d'água até vinte metros.

\section{Analisador de Gases}

Modelo TEEM 100, para medida direta do $\mathrm{VO}_{2}$ (Figura 6). Este aparelho coleta amostras do gás expirado através de um pneumotógrafo que é acoplado num bocal, o qual é colocado no indivíduo. Utiliza-se um oclusor nasal, obrigando o indivíduo a respirar apenas pela boca.

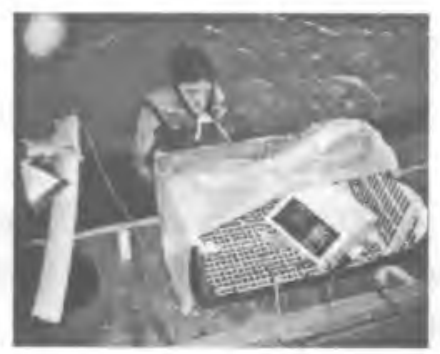

Figura 6 - Aparelho para coleta e análise de gases

\section{$\underline{\text { Lactímetro }}$}

Para a determinação dos níveis de lactato sangüíneo utilizou-se dois analisadores portáteis da marca Böhering, ACUSPORT ${ }^{\mathrm{TM}}$ (portable blood lactate analyser - Boehringer Manhein/ Manhein) com fitas da mesma marca e micro lancetas esterilizadas (SoftClik) em "caneta".

\section{Metrônomo}

Para a determinação da frequiência de execução dos exercícios foi utilizado um metrônomo WITTNER modelo Taktell Júnior.

\section{Cronômetros}

Foram utilizados dois cronômetros da marca Cassio, com resolução de décimo de segundo; sendo um cronômetro oficial e outro reserva para possíveis imprevișţ̣s. Os cronômetros foram utilizados para o controle do tempo durante a realização dos exercícios físicos.

\section{Testagem dos instrumentos}

Ficha de Dados Individuais.

A ficha de dados individuais foi testada por três professores de Educação Física, especialistas em Fisiologia do Exercício, quanto à sua objetividade e validade.

\section{Plataformas de Forca}

Com a intenção de automatizar o processo de calibração com a ajuda das macros do programa SAD (macros são seqüências de operações que o programa executa automaticamente), os ensaios foram padronizados da seguinte forma: um tempo inicial, sem qualquer carga sobre a Revista Kinesis, Santa Maria, $N^{\circ}$ Especial, p. 110-154, nov. 2001 
plataforma, para avaliação do zero de cada canal; após, sequiências de patamares de carga com tempo fixo, dividido em uma parte para o acerto da carga e outra sem qualquer modificação de carga, para avaliação das médias das cargas deste patamar.

Assim, nos ensaios de componente de força foi seguido o seguinte protocolo:

- 40 segundos de repouso para avaliação do zero do ensaio;

- 10 segundos para acerto do primeiro patamar de força em 19,777 N;

- 40 segundos de repouso para avaliação da média deste patamar;

- repetição dos dois últimos passos até a carga de 276,877 N; e

- descarregamento e repouso de 40 segundos para nova avaliação do zero.

Balança

A balança foi aferida com pesos padrões antes de iniciar o experimento.

\section{Variáveis}

Variáveis Dependentes

- Frequiência Cardíaca

- Consumo de Oxigênio

- Concentração de Lactato

- Força de Reação Vertical (pico máximo)

- Impulso

Variáveis Independentes

- Exercícios (Figura 7) fora d'água e em diferentes profundidades de água (neste estudo foram utilizados as profundidades de água de cicatriz umbilical e de ombro).

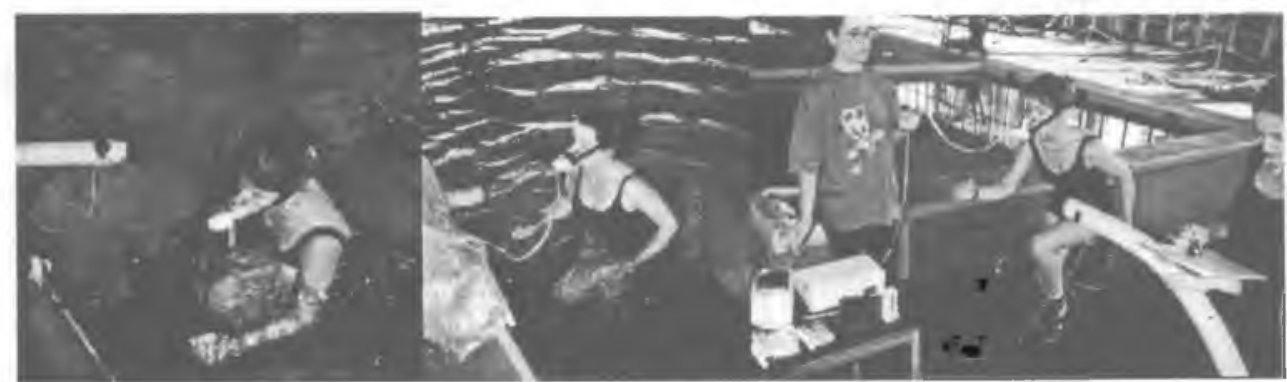

Figura 7 - Exercícios nas profundidades de ombro, cîcatriz umbilical e fora d'água

Variáveis de Controle

- Temperatura da água: neste estudo oscilou entre 30 e $31^{\circ} \mathrm{C}$;

\section{Desenho experimental}

Neste estudo foram utilizados cinco grupos (um grupo para cada exercício) experimentais (Quadro 1) 
Quadro 1 - Desenho experimental da amostra

\begin{tabular}{|c|c|c|}
\hline GRUPOS & TRATAMENTOS & TESTES \\
\hline \multirow{3}{*}{$\mathrm{A}$} & $\mathrm{X}_{1}$ & $\mathrm{O}_{1}-\mathrm{O}_{2}-\mathrm{O}_{3}$ \\
& $\mathrm{X}_{2}$ & $\mathrm{O}_{1}-\mathrm{O}_{2}-\mathrm{O}_{3}$ \\
& $\mathrm{X}_{3}$ & $\mathrm{O}_{1}-\mathrm{O}_{2}-\mathrm{O}_{3}$ \\
\hline \multirow{3}{*}{$\mathrm{B}$} & $\mathrm{X}_{1}$ & $\mathrm{O}_{1}-\mathrm{O}_{2}-\mathrm{O}_{3}$ \\
& $\mathrm{X}_{2}$ & $\mathrm{O}_{1}-\mathrm{O}_{2}-\mathrm{O}_{3}$ \\
\hline & $\mathrm{X}_{3}$ & $\mathrm{O}_{1}-\mathrm{O}_{2}-\mathrm{O}_{3}$ \\
\hline $\mathrm{C}$ & $\mathrm{X}_{1}$ & $\mathrm{O}_{1}-\mathrm{O}_{2}-\mathrm{O}_{3}$ \\
& $\mathrm{X}_{2}$ & $\mathrm{O}_{1}-\mathrm{O}_{2}-\mathrm{O}_{3}$ \\
& $\mathrm{X}_{3}$ & $\mathrm{O}_{1}-\mathrm{O}_{2}-\mathrm{O}_{3}$ \\
\hline & $\mathrm{X}_{1}$ & $\mathrm{O}_{1}-\mathrm{O}_{2}-\mathrm{O}_{3}$ \\
$\mathrm{D}$ & $\mathrm{X}_{2}$ & $\mathrm{O}_{1}-\mathrm{O}_{2}-\mathrm{O}_{3}$ \\
& $\mathrm{X}_{3}$ & $\mathrm{O}_{1}-\mathrm{O}_{2}-\mathrm{O}_{3}$ \\
\hline \multirow{2}{*}{$\mathrm{E}$} & $\mathrm{X}_{1}$ & $\mathrm{O}_{1}-\mathrm{O}_{2}-\mathrm{O}_{3}$ \\
& $\mathrm{X}_{2}$ & $\mathrm{O}_{1}-\mathrm{O}_{2}-\mathrm{O}_{3}$ \\
& $\mathrm{X}_{3}$ & $\mathrm{O}_{1}-\mathrm{O}_{2}-\mathrm{O}_{3}$ \\
\hline
\end{tabular}

A = grupo experimental executando o exercício Garça (FIG. 08);

$\mathrm{B}=$ grupo experimental executando o exercício Lagosta (FIG. 01);

$\mathrm{C}=$ grupo experimental executando o exercício Jacaré I (FIG. 10);

$\mathrm{D}$ = grupo experimental executando o exercício Jacaré II (FIG. 04);

$\mathrm{E}$ = grupo experimental executando o exercício Pelicano (FIG. 05);

$\mathrm{O}_{1}=$ medidas de repouso, que ocorreram antes das sessões de treinamento das variáveis: $\mathrm{FC}, \mathrm{VO}_{2}$, LACTATO;

$\mathrm{O}_{2}=$ medidas que ocorreram durante as sessões de treinamento das variáveis: $\mathrm{FC}, \mathrm{VO}_{2}$, FORÇAS DE REAÇÃO VERTICAIS e de IMPULSO;

$\mathrm{O}_{3}=$ medidas de recuperação, que foram realizadas após as sessões de treinamento das variáveis: FC e LACTATO;

$\mathrm{X}_{1}=$ tratamento 1 , os indivíduos realizavam o exercício na profundidade de água ao nível de ombro;

$\mathrm{X}_{2}=$ tratamento 2 , os indivíduos realizavam o exercício na profundidade de água ao nível de cicatriz umbilical;

$\mathrm{X}_{3}=$ tratamento 3 , os indivíduos realizavam o exercício fora d'água.

\section{Procedimentos da coleta de dados}

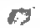

Foram utilizadas as dependências do Centro Natatório Frederico Guilherme Gaelzer e do LAPEX da ESEF-UFRGS, para a coleta de dados.

Os indivíduos selecionados compareceram ao Centro Natatório em dias e horários prédeterminados.

Para a coleta dos dados da amostra foram sorteados cinco exercícios de hidroginástica dos dez exercícios mais utilizados pelos professores de hidroginástica do Brasil (exercícios estes determinádos por Moraes, 1998). Cada exercício deveria ser executado por 5 indivíduos diferentes, fora d'água e nas profundidades de água de cicatriz umbilical e ombro, durante 5 minutos, na frequiência média de execução

Revista Kinesis, Santa Maria, $N^{o}$ Especial, p. 112-154, nov. 2001 
do exercício determinada por Moraes (1998), na segunda etapa de seu estudo. O indivíduo realizava o exercício sorteado, primeiramente, durante 15 segundos, com o objetivo de adaptação, ao final dos 15 segundos era feita a contagem do número médio de repetições. Quando este número não correspondeu à média, proposta pela referida autora, foi considerado o número médio de repetições com um desvio padrão acima, com o objetivo de fazer com que o indivíduo se aproximasse da realidade de uma aula de hidroginástica com uma sensação subjetiva ao esforço de nível moderado.

O tempo de recuperação entre um exercício e outro foi determinado de forma individual e dependeu do tempo que o indivíduo levou para que seu lactato e a sua FC voltassem aos níveis de repouso, até o tempo máximo de 50 minutos. Caso neste tempo o indivíduo não tivesse retornado ao níveis de repouso, o mesmo realizava a série seguinte. A ordem de execução das séries foi primeiramente com água na altura do ombro, depois com água na altura da cicatriz umbilical e por último fora d'água.

Os exercícios sorteados foram o Garça I (Figura 4), Lagosta I (Figura 1), Jacaré I (Figura 5) e II (Figura 2) e o Pelicano (Figura 3).

Para as medidas fisiológicas e biomecânicas as mulheres deveriam estar em trajes de banho (maiô ou biquíni) e descalças.

Primeiramente, foi feita a leitura da estatura e do peso corporal. Após o indivíduo deveria permanecer em repouso, sentado, por no mínimo 10 minutos para a coleta dos valores de FC, $\mathrm{VO}_{2}$ e LACTATO de repouso.

Após as coletas de repouso, cada indivíduo foi submetido ao exercício de hidroginástica previamente sorteado. $\mathrm{O}$ exercício foi executado durante 5 minutos, e durante o mesmo foi feito:

1) o registro da $\mathrm{FC}$ de um em um minuto;

2) a análise do $\mathrm{VO}_{2}$ de vinte em vinte 20 segundos; e

3) o registro das forças de reação verticais durante os dois primeiros minutos de exercício.

Ao término do exercício foi feito o registro:

1) da FC de um em um minuto até o vigésimo minuto, e após caso fosse necessário, de cinco em cinco minutos;

2) da concentração de lactato sangüíneo no terceiro minuto, no quinto minuto e de cinco em cinco minutos até que a concentração de lactato voltasse aos níveis de repouso; e

3) do $\mathrm{VO}_{2}$, que foi coletado imediatamente após o término»do exercício.

\section{Rotinas para determinação das variáveis dependentes}

Rotina para determinação do Consumo de Oxigênio: para determinação do Consumo de Oxigênio foi feita a leitura do consumo de oxigênio de repouso (sentado fora d'água) $e$ durante o exercício, de vinte em vinte segundos até o término de cada série de exercício.

A coleta do $\mathrm{VO}_{2}$ foi feita através de medida direta utilizando o aparelho portatil modelo TEEM 100, o mesmo foi colocado na borda da piscina (Figura 12, página 63) dentro de uma cesta plastica isolada por um saco plastico.

Rotina para determinação da concentração de lactato sangüíneo: para avaliação nas alterações dos níveis de lactato sangüíneo os indivíduos submeteram-se à coleta de sangue: a) 
antes do inicio do teste, e b) após cada série de exercício no terceiro minuto, no quinto minuto e de cinco em cinco minutos até que a concentração de lactato voltasse aos níveis de repouso.

A dosagem do lactato foi realizada com o indivíduo sentado no lado de fora da piscina. A proximidade viabilizou as coletas logo ao término de cada série. A coleta foi através do aparelho portátil modelo ACCUSPORT ${ }^{\mathrm{TM}}$. Optou-se por coletar o sangue no dedo indicador e utilizou-se compressas de gaze hidrófila embebidas em álcool para assepsia do local.

Rotina para determinação da frequêencia cardíaca: para a leitura da FCR o indivíduo permaneceu sentado, por no mínimo 10 minutos, após foi feita a leitura da FCR. A leitura da FC durante o exercício foi feita de um em um minuto. A leitura da FC durante o intervalo entre as séries foi realizada de um em um minuto até o vigésimo minuto. Caso fosse necessário continuar fazendo a leitura da FC após este tempo a mesma foi coletada de cinco em cinco minutos até que o indivíduo atingisse novamente a FCR.

Rotina para determinação das forças de reação na vertical; os ensaios foram executados todos em uma seqüência pré-estabelecida. Foi separado o canal da força na direção vertical $(\mathrm{Fz})$ das plataformas. Conforme Roesler (1997), através da transformada de Fourier as frequiências dos exercícios realizados dentro d'água em seu experimento estavam contidas na faixa de 0 a 6 $\mathrm{Hz}$, sendo que o pico que se observa em $60 \mathrm{~Hz}$ é causado pelo ruído captado da rede elétrica. Considerando estes resultados utilizou-se nesta pesquisa uma filtragem com filtro passa-baixo com frequeuencia de corte de $10 \mathrm{~Hz}$ como margem de segurança. Após foi feita uma média dos picos de força obtidos por cada indivíduo em cada série, sendo esta média considerada o pico de força na vertical executada pelo indivíduo em uma série.

Rotina para determinação do impulso: como o impulso, segundo Hay (1981), é igual à área sob a curva força-tempo para um dado intervalo de tempo, e o impulso total é a soma de todos os números infinitos de tais impulsos menores, em nosso trabalho o impulso total foi calculado matematicamente como sendo a integral da curva força-tempo no intervalo de tempo de execução de uma série do exercício dividido pelo número de repetições realizadas.

\section{Tratamento estatístico}

Foi utilizada a estatística descritiva, o teste de normalidade de Shapiro-Wilk, a análise de variância ANOVA e teste F, para comparar as classes de variáveis classificatórias. Para a localização das diferenças, utilizou-se o teste de Tukey. Utilizou-se tanłbém a Análise Fatorial de Componentes Principais, a Correlação Linear do Produto Momento-de Pearson para verificar a correlação entre as variáveis fisiológicas e biomecânicas e a Análise de Regressão Linear para determinarmos o grau de influência de uma variável sobre outra. O pacote estatístico computacional utilizado foi o SPSS for Windows, versão 8.0.

\section{Resultados e discussão}

Inicialmente, com a finalidade de caracterizar a amostra, apresenta-se a Tabela 1 com os resultados médios e desvios padrões das variáveis: idade (ID), estatura (EST), peso corporal total
(PCT) dos indivíduos.

Revista Kinesis, Santa Maria, $N^{o}$ Especial, p. 114-154, nov. 2001 
Tabela 1 - Médias, desvio padrão, erro padrão, intervalo de confiança da média e valores mínimos e máximos das variáveis peso, estatura e idade

\begin{tabular}{lrrrrrrr}
\hline $\begin{array}{c}\text { Variáveis } \\
\mathrm{N}=23\end{array}$ & \multicolumn{1}{c}{$\chi$} & $\delta$ & Erro Padrão & \multicolumn{2}{c}{$\begin{array}{l}\text { 95\% do Intervalo de } \\
\text { Confiança da Média }\end{array}$} & Mínimo & Máximo \\
& & & & \multicolumn{7}{c}{$\begin{array}{l}\text { Limite } \\
\text { inferior }\end{array}$} & $\begin{array}{c}\text { Limite } \\
\text { superior }\end{array}$ \\
\hline PESO (kgf) & 66,07 & $\pm 8,87$ & 1,8488 & 62,2397 & 69,9081 & 50,00 & 83,3 \\
ESTATURA (cm) & 156,15 & $\pm 8,86$ & 1,8489 & 152,3135 & 159,9821 & 147,50 & 171,0 \\
IDADE (anos) & 54,00 & $\pm 11,16$ & 2,3270 & 49,1741 & 58,8259 & 39,00 & 71,0 \\
\hline
\end{tabular}

Para testarmos a normalidade da amostra utilizou-se o teste de Shapiro-Wilk, o qual é indicado para amostras com menos de 50 indivíduos. Os resultados (Tabela 2) apresentaram uma distribuição normal.

Tabela 2 - Teste de Normalidade da amostra. Variáveis $\mathrm{VO}_{2} \mathrm{O}, \mathrm{VO}_{2} \mathrm{U}, \mathrm{VO}_{2} \mathrm{~F} F C O, F C U, F C F$, ImpO, ImpU, ImpF, FzF, FzU, FzO, LacO, LacU, LacF.

\begin{tabular}{cc}
\hline Variáveis e condições experimentais & Shapiro-Wilk \\
\hline $\mathrm{VO}_{2} \mathrm{~F}$ &, 393 \\
$\mathrm{VO}_{2} \mathrm{U}$ &, 384 \\
$\mathrm{VO}_{2} \mathrm{O}$ &, 988 \\
$\mathrm{FCF}$ &, 981 \\
$\mathrm{FCU}$ &, 278 \\
$\mathrm{FCO}$ &, 252 \\
$\mathrm{ImpF}$ &, 868 \\
$\mathrm{ImpU}$ &, 967 \\
$\mathrm{ImpO}$ &, 652 \\
$\mathrm{FzF}$ &, 424 \\
$\mathrm{FzU}$ &, 881 \\
$\mathrm{FzO}$ &, 907 \\
$\mathrm{LacF}$ &, 192 \\
$\mathrm{LacU}$ &, 727 \\
$\mathrm{LacO}$ &, 565 \\
\hline
\end{tabular}

* diferença estatisticamente significante

Um dos objetivos específicos desse estudo foi determinar e comparar o comportamento das variáveis fisiológicas ( $\mathrm{FCO}, \mathrm{FCU}, \mathrm{FCF}, \mathrm{LacO}, \mathrm{LacU}, \mathrm{LacF}, \mathrm{VO}_{2} \mathrm{O}, \mathrm{VO}_{2} \mathrm{U}$ e $\mathrm{VO}_{2} \mathrm{~F}$ ) e biomecânicas (ImpO, ImpU, ImpF, FzO, FzU e FzF) entre cinco exercícios de hidroginástica (Garça, Lagosta, Jacaré I, Jacaré II e Pelicano).

Na comparação das médias das variáveis analisadas entre os cinco exercícios propostos utilizou-se a análise de variância, ao nível de significância de 5\% (Tabela 3), e com a intenção de verificar em quais variáveis e exercícios encontravam-se as diferenças entre as médias das variáveis estudadas empregou-se o teste post-hoc de Tukey. 
Tabela 3 - Médias, desvio padrão e freqüência de execução $(f)$ dos exercícios nas variáveis FCO, FCU, FCF, LacO, LacU, LacF, $\mathrm{VO}_{2} \mathrm{O}, \mathrm{VO}_{2} \mathrm{U}, \mathrm{VO}_{2} \mathrm{~F}, \mathrm{ImpO}, \mathrm{ImpU}, \mathrm{ImpF}, \mathrm{FzO}, \mathrm{FzU}, \mathrm{FzF}$ e análise de variança entre os exercícios.

\begin{tabular}{|c|c|c|c|c|c|c|c|c|c|c|}
\hline & \multicolumn{2}{|c|}{$\begin{array}{c}\text { Garça }(n=4) \\
f=57 \pm 7 \mathrm{rep} / \mathrm{min}\end{array}$} & \multicolumn{2}{|c|}{$\begin{array}{c}\text { Lagosta }(\mathrm{n}=5) \\
f=86 \pm 21 \mathrm{rep} / \mathrm{min}\end{array}$} & \multicolumn{2}{|c|}{ Jacaré I $(n=5)$} & \multicolumn{2}{|c|}{ Jacaré II $(n=5)$} & \multicolumn{2}{|c|}{ Pelicano $(n=4)$} \\
\hline & $\chi$ & $\delta$ & $\chi$ & $\delta$ & $\chi$ & $\delta$ & $\chi$ & $\delta$ & $\chi$ & $\delta$ \\
\hline FCO & 120,3 & $\pm 17,06$ & 118,5 & $\pm 4,07$ & 127,2 & \pm 12 & 120,3 & $\pm 5,38$ & 130,0 & $\pm 15,08$ \\
\hline $\mathrm{FCU}$ & 132,5 & $\pm 19,49$ & 123,8 & $\pm 18,51$ & 138,8 & $\pm 11,82$ & 145,8 & $\pm 27,51$ & 135,0 & $\pm 14,76$ \\
\hline FCF & 132,0 & $\pm 22,08$ & 126,8 & $\pm 17,37$ & 144,8 & $\pm 21,21$ & 158,6 & $\pm 20,72$ & 159,8 & $\pm 21,76$ \\
\hline $\mathrm{LacO}$ & 2,3 & $\pm 0,64$ & 1,6 & $\pm 0,21$ & 1,9 & $\pm 0,73$ & 1,7 & $\pm 0,35$ & 2,4 & $\pm 0,54$ \\
\hline $\mathrm{LacU}$ & 2,6 & $\pm 0,72$ & 2,0 & $\pm 0,42$ & 2,1 & $\pm 0,54$ & 2,2 & $\pm 0,20$ & 2,3 & $\pm 0,61$ \\
\hline $\mathrm{LacF}$ & 3,3 & $\pm 1,56$ & 3,2 & $\pm 0,95$ & 3,1 & $\pm 1,32$ & 4,8 & $\pm 1,69$ & 4,6 & $\pm 1,59$ \\
\hline $\mathrm{VO}_{2} \mathrm{O}$ & 15,3 & $\pm 2,23$ & $12,8 *$ & $\pm 2,07$ & 16,9 & $\pm 1,59$ & 14,3 & $\pm 3,77$ & $18,3^{*}$ & $\pm 2,91$ \\
\hline $\mathrm{VO}_{2} \mathrm{U}$ & 16,6 & $\pm 2,66$ & 16,3 & $\pm 1,60$ & 20,5 & $\pm 2,92$ & 18,5 & $\pm 3,74$ & 19,1 & $\pm 2,37$ \\
\hline $\mathrm{VO}_{2} \mathrm{~F}$ & $15,4^{*}$ & $\pm 4,19$ & 17,9 & $\pm 1,21$ & 21,6 & $\pm 2,92$ & 23,3 & $\pm 6,91$ & $25,0^{*}$ & $\pm 4,45$ \\
\hline ImpO & 16,9 & $\pm 3,28$ & 12,9 & $\pm 3,28$ & 12,8 & $\pm 2,72$ & 11,9 & $\pm 3,27$ & 14,5 & $\pm 3,40$ \\
\hline$[\mathrm{mpU}$ & 19,4 & $\pm 5,23$ & 17,2 & $\pm 4,50$ & 18,5 & $\pm 2,40$ & 16,1 & $\pm 3,30$ & 21,0 & $\pm 5,28$ \\
\hline ImpF & 78,3 & $\pm 30,22$ & 57,7 & $\pm 17,63$ & 57,1 & $\pm 26,43$ & 55,8 & $\pm 10,66$ & 65,2 & $\pm 21,89$ \\
\hline $\mathrm{FzO}$ & 40,75 & $\pm 7,72$ & 52,01 & $\pm 19,82$ & 56,88 & $\pm 14,06$ & 48,71 & $\pm 13,27$ & 48,43 & $\pm 8,84$ \\
\hline $\mathrm{FzU}$ & 47,80 & $\pm 19,90$ & 66,36 & $\pm 23,20$ & 62,39 & $\pm 16,80$ & 65,14 & $\pm 18,42$ & 58,02 & $\pm 12,11$ \\
\hline $\mathrm{FzF}$ & 175,27 & $\pm 74,28$ & 193,99 & $\pm 74,73$ & $\begin{array}{r}199,8 \\
3\end{array}$ & $\pm 72,52$ & 242,98 & $\pm 47,36$ & 202,28 & $\pm 86,15$ \\
\hline
\end{tabular}

* diferença estatisticamente significante $\mathrm{p}<0.05$

Os resultados (Tabela 3) demonstraram não haver diferenças estatisticamente significantes entre as variáveis analisadas, com exceção do consumo de oxigênio na profundidade de ombro entre o exercício 2 (Lagosta) e o exercício 5 (Pelicano) e do consumo de oxigênio fora d'água entre o exercício 1 (Garça) e o exercício 5 (Pelicano).

Por este motivo para analisarmos se existem diferenças nas variáveis entre as diferentes profundidades de água e os exercícios realizados fora d'água, considerou-se não existir diferenças estatisticamente significantes entre os exercícios propostos, e a análise dos resultados foi realizada com todos os exercícios reunidos em um só grupo.

Já, para a comparação das médias das variáveis analisadas entre as duas profundidades de água propostas e a realização do exercício fora d'água utilizou-se a análise de variância (Tabela 4). Os valores de F encontrados permitem afirmar que as diferenças encontradas entre os tratamentos foram altamente significantes.

Da mesma forma para verificar em quais tratamentos encontravam-se as diferenças entre as médias das variáveis estudadas também foi empregado o teste post-hoc de Tukey (Tabela 5).

O comportamento de todas as variáveis estudadas na execução dos exercícios demonstrou semelhança quanto à diminuição progressiva de seus valores, desde o tratamento fora d'água até a maior profundidade de imersão. 
Tabela 4 - Análise de Variança das variáveis $\mathrm{VO}_{2}$, FC, Lac, Imp e Fz nos diferentes tratamentos (fora d'água e nas profundidades de água de cicatriz umbilical e ombro)

\begin{tabular}{|c|c|c|c|c|c|c|}
\hline & & $\begin{array}{c}\text { Soma dos } \\
\text { Quadrados } \\
\text { (SQ) }\end{array}$ & $\begin{array}{c}\text { Graus de } \\
\text { Liberdade (GL) }\end{array}$ & $\begin{array}{l}\text { Quadrado Médio } \\
\text { (QM) }\end{array}$ & $\mathrm{F}$ & Sig. \\
\hline \multirow[t]{3}{*}{$\mathrm{VO}_{2}$} & Entre Tratamentos & 322,475 & 2 & 161,238 & 10,523 &, 000 \\
\hline & Erro (intratratamentos) & 1011,275 & 66 & 15,322 & & \\
\hline & Total & 1333,750 & 68 & & & \\
\hline \multirow[t]{3}{*}{$\mathrm{FC}$} & Entre Tratamentos & 7216,899 & 2 & 3608,449 & 9,057 &, 000 \\
\hline & Erro (intratratamentos) & 26295,043 & 66 & 398,410 & & \\
\hline & Total & 33511,942 & 68 & & & \\
\hline \multirow[t]{3}{*}{ Lac } & Entre Tratamentos & 43,215 & 2 & 21,608 & 22,760 &, 000 \\
\hline & Erro (intratratamentos) & 61,709 & 65 & ,949 & & \\
\hline & Total & 104,925 & 67 & & & \\
\hline \multirow[t]{3}{*}{$\operatorname{Imp}$} & Entre Tratamentos & 32777,803 & 2 & 16388,902 & 100,149 &, 000 \\
\hline & Erro (intratratamentos) & 10800,617 & 66 & 163,646 & & \\
\hline & Total & 43578,421 & 68 & & & \\
\hline \multirow[t]{3}{*}{$\mathrm{Fz}$} & Entre Tratamentos & 341448,485 & 2 & 170724,242 & 98,847 &, 000 \\
\hline & Erro (intratratamentos) & 113991,885 & 66 & 1727,150 & & \\
\hline & Total & 455440,369 & 68 & & & \\
\hline
\end{tabular}

Os resultados do $\mathrm{VO}_{2}$ encontrados (Tabela 5) demonstraram não existir diferenças estatisticamente significantes entre o exercício realizado fora d'água $(20,69 \pm 5,25 \mathrm{ml} . \mathrm{kg}$ $\left.{ }^{1} \cdot \mathrm{min}^{-1}\right)$ e o exercício realizado na profundidade de cicatriz umbilical $\left(18,22 \pm 2,99 \mathrm{ml} . \mathrm{kg}^{-}\right.$ $\left.{ }^{1} \cdot \mathrm{min}^{-1}\right)$. Entretanto ao imergirmos mais o indivíduo até a profundidade de ombro $(15,40 \pm 3,07$ $\mathrm{ml} . \mathrm{kg}^{-1} \cdot \mathrm{min}^{-1}$ ) encontramos diferenças estatisticamente significantes em relação aos exercícios executados fora d'água e na profundidade de cicatriz umbilical. Os resultados demonstraram que conforme aumentava a profundidade de imersão diminuía o consumo de oxigênio.

Os indivíduos que se exercitavam na profundidade de cicatriz umbilical demonstraram uma diminuição de $11,94 \%$ no $\mathrm{VO}_{2}$ em relação aos exercícios realizados fora d'água; e na profundidade de ombro a diminuição foi de $25,57 \%$.

Estes resultados demonstram que um indivíduo ao realizar um exercício numa cadência e em diferentes profundidades, tem o seu gasto energético diminuído à medida que aumenta o nível de imersão. Tais resultados confirmam os estudos de Costill (1971), Avellini et al. (1983), Vickery et al. (1983), Gleim \& Nicholas (1989) e Town \& Bradley"(1991).

O comportamento da FC (Tabela 12) foi semelhante ǻ comportamento do $\mathrm{VO}_{2}$, pois os resultados demonstraram não haver diferença estatisticamente significante entre a FCF $(144,26 \pm 23,08)$ e a FCU $(135,30 \pm 19,14)$, e haver uma diferença estatisticamente significante entre os tratamentos citados e o comportamento da FCO $(119,52 \pm 17,22)$.

Os indivíduos que se exercitavam na profundidade de cicatriz umbilical demonstraram uma diminuição de $6,25 \%$ na $\mathrm{FC}$ em relação aos exercícios realizados fora d'água; enquanto que na profundidade de ombro a diminuição foi de $17,36 \%$.

Ao compararmos a diferença encontrada na FC entre o exercício fora d'água e nas profundidades de cicatriz umbilical e ombro, encontramos um decréscimo de 9 bpm e 23 bpm 
respectivamente. Kruel (1994), com indivíduos estáticos, encontrou um decréscimo de 12 bpm (FCU) e 16 bpm (FCO). Kruel (1997), noutro estudo, também com os indivíduos estáticos, encontrou um decréscimo de $11 \mathrm{bpm}$ (FCU) e $13 \mathrm{bpm}$ (FCO). Os dados do presente estudo e os da literatura demonstram que a diminuição de FC ocorre tanto em movimento como quando os indivíduos estão estáticos no meio líquido, sendo maior o nível de decréscimo quando os indivíduos estão em movimento.

Tabela 5 - Valores médios, desvios padrões, erro, intervalo de confiança e valores máximos e mínimos das variáveis FCO, FCU, FCF, LacO, LacU, $\mathrm{LacF}, \mathrm{VO}_{2} \mathrm{O}, \mathrm{VO}_{2} \mathrm{U}, \mathrm{VO}_{2} \mathrm{~F}, \mathrm{ImpO}, \mathrm{ImpU}$, $\mathrm{ImpF}, \mathrm{FzO}, \mathrm{FzU}$ e FzF

\begin{tabular}{lcrrrrrrr}
\hline Variáveis & $\mathrm{N}$ & $\mathrm{X}$ & $\delta$ & $\begin{array}{c}\text { Erro } \\
\text { Padrão }\end{array}$ & $\begin{array}{c}\text { 95\% do Intervalo de Confiança } \\
\text { da Média }\end{array}$ & Mínimo & Máximo \\
\hline & & & \multicolumn{7}{c}{ Limite inferior Limite superior } \\
\hline $\mathrm{VO}_{2} \mathrm{~F}$ & 23 & $20,69^{\mathrm{a}}$ & $\pm 5,25$ & 1,0956 & 18,42 & 22,96 & 10,60 & 32,10 \\
$\mathrm{VO}_{2} \mathrm{U}$ & 23 & $18,22^{\mathrm{a}}$ & $\pm 2,99$ & 0,6238 & 16,93 & 19,52 & 13,70 & 24,40 \\
$\mathrm{VO}_{2} \mathrm{O}$ & 23 & $15,40^{\mathrm{b}}$ & $\pm 3,07$ & 0,6396 & 14,07 & 16,72 & 9,30 & 21,60 \\
\hline $\mathrm{FCF}$ & 23 & $144,26^{\mathrm{a}}$ & $\pm 23,08$ & 4,8128 & 134,28 & 154,24 & 98,00 & 190,00 \\
$\mathrm{FCU}$ & 23 & $135,30^{\mathrm{a}}$ & $\pm 19,14$ & 3,9898 & 127,03 & 143,58 & 91,00 & 189,00 \\
$\mathrm{FCO}$ & 23 & $119,52^{\mathrm{b}}$ & $\pm 17,22$ & 3,5895 & 112,08 & 126,97 & 78,00 & 147,00 \\
\hline $\mathrm{LacF}$ & 23 & $3,77^{\mathrm{a}}$ & $\pm 1,50$ & 0,3134 & 3,12 & 4,42 & 1,70 & 6,70 \\
$\mathrm{LacU}$ & 22 & $2,22^{\mathrm{b}}$ & $\pm 0,48$ & 0,1025 & 2,01 & 2,43 & 1,40 & 3,40 \\
$\mathrm{LacO}$ & 23 & $1,97^{\mathrm{b}}$ & $\pm 0,57$ & 0,1188 & 1,73 & 2,22 & 1,10 & 3,10 \\
\hline $\mathrm{ImpF}$ & 23 & $62,02^{\mathrm{a}}$ & $\pm 21,50$ & 4,4838 & 52,73 & 71,32 & 20,20 & 101,82 \\
$\mathrm{ImpU}$ & 23 & $18,29^{\mathrm{b}}$ & $\pm 4,14$ & 0,8639 & 16,50 & 20,09 & 9,77 & 27,08 \\
$\mathrm{ImpO}$ & 23 & $13,64^{\mathrm{b}}$ & $\pm 3,37$ & 0,7029 & 12,18 & 15,10 & 7,32 & 21,38 \\
\hline FzF & 23 & $204,09^{\mathrm{a}}$ & $\pm 68,31$ & 14,2440 & 174,55 & 233,63 & 80,67 & 314,49 \\
FzU & 23 & $60,55^{\mathrm{b}}$ & $\pm 18,13$ & 3,7810 & 52,71 & 68,39 & 19,92 & 93,44 \\
FzO & 23 & $49,77^{\mathrm{b}}$ & $\pm 13,64$ & 2,8446 & 43,87 & 55,67 & 20,68 & 75,09 \\
\hline
\end{tabular}

Obs. Letras diferentes indicam diferenças estatisticamente significantes para um $p<0,05$

Os resultados encontrados corroboram com os estudos realizados por Heigenhauser et al. (1977), Sheldahl et al. (1984), Hamer \& Morton (1990) e Town \& Bradley (1991). De acordo com os autores citados anteriormente, a significante redução da FC ocorre porque, durante os exercícios na água, os indivíduos podem estar se exercitando com męonos força cardiovascular. Isto poderia ser devido a uma eficiência maior do coração como uma bomba, associada a um
aumento no volume de batimentos e FC diminuídas.

No nosso entendimento esta diminuição de FC está associada a uma diminuição no peso hidrostático do indivíduo quando o mesmo está imerso no meio líquido, pois de acordo com Kruel (1994) e Kruel (1995a) ocorre, em mulheres, uma redução no peso hidrostático de $70 \%$ na profundidade da cicatriz umbilical e de $85 \%$ na profundidade do ombro, fazendo, provavelmente, com que menos unidades motoras sejam recrutadas para realizar movimento no sentido vertical, com conseqüente gasto energético menor. Entretanto, mesmo não tendo sido objeto deste estudo, quando os indivíduos se deslocam no sentido horizontal, mais unidades motoras devem ser recrutadas para vencer a maior resistência que a água oferece em relação ao ar.

Revista Kinesis, Santa Maria, $N^{o}$ Especial, p. 118-154, nov. 2001 
Provavelmente, pelo motivo citado anteriormente, os resultados encontrados nesse estudo não coincidem com os demonstrados por Lazzari \& Meyer (1997) onde os autores encontraram um aumento na $\mathrm{FC}$ e no $\mathrm{VO}_{2}$ quando os exercícios são realizados na água com um deslocamento na horizontal (corrida).

$\mathrm{Na}$ literatura encontramos também resultados contraditórios, pois enquanto Johnson et al. (1977) encontraram um aumento do $\mathrm{VO}_{2}$ e da $\mathrm{FC}$ dentro d'água, Cassady \& Nielsen (1992), relatam um aumento do $\mathrm{VO}_{2}$ e uma diminuição da $\mathrm{FC}$, enquanto os dados do presente estudo demonstram uma diminuição do $\mathrm{VO}_{2}$, da $\mathrm{FC}$ bem como da concentração de lactato sangüíneo.

A variável lactato (Tabela 5) apresentou um comportamento diferente das variáveis $\mathrm{VO}_{2}$ e $\mathrm{FC}$, pois não foram encontradas diferenças estatisticamente significantes entre o LacO $(1,97 \pm 0,57)$ e o $\operatorname{LacU}(2,22 \pm 0,48)$. Entretanto os exercícios realizados dentro d'água apresentaram uma diferença estatisticamente significante em relação aos exercícios realizados fora d'água $(3,77 \pm 1,50)$, comportamento este semelhante ao apresentado pelas variáveis biomecânicas (Fz e Imp).

Os valores de lactato encontrados nas profundidades de cicatriz umbilical e ombro mostraram uma redução de $41,12 \%$ e $47,75 \%$, respectivamente, em relação aos valores encontrados fora d'água. Town \& Bradley (1991) encontraram uma redução de apenas $19 \%$ nas concentrações de lactato no meio líquido, provavelmente porque os indivíduos de sua amostra realizaram exercícios com deslocamento horizontal.

As duas variáveis biomecânicas estudadas (Tabela 5) apresentaram um comportamento semelhante, não tendo sido encontradas diferenças estatisticamente significantes entre os tratamentos realizados nas diferentes profundidades de água, mas demonstrando uma diferença estatisticamente significante entre o tratamento realizado fora d'água quando comparado com os tratamentos realizados dentro d'água.

Os resultados de $\mathrm{FzO}$ e $\mathrm{FzU}$ foram, respectivamente 4,1 e 3,37 vezes menores do que os resultados de FzF (Figura 8), demonstrando claramente que os exercícios dentro d'água, quando executados com o objetivo de proteção e preservação das articulações, podem e devem ser prescritos.

Os resultados de ImpO e ImpU foram, respectivamente 4,55 e 3,39 vezes menores do que os resultados de ImpF (Tabela 12).

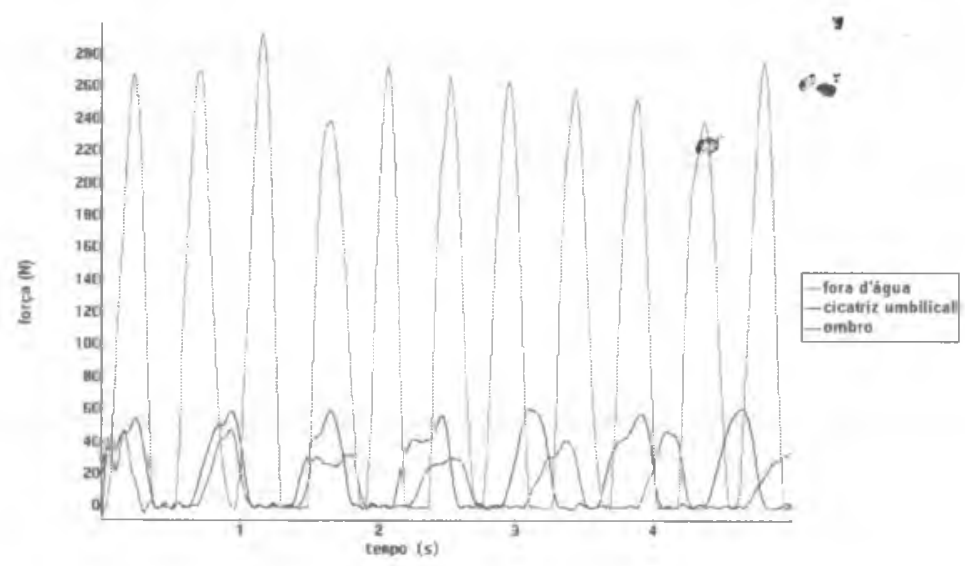

Figura 8 - Gráfico representativo da Fz nos diferentes tratamentos 
Com o objetivo de reduzir o número de variáveis em dimensões para melhor explicar a variabilidade do fenômeno analisado, utilizou-se a análise fatorial de componentes principais (Tabela 6, Tabela 8 e Tabela 10) nos diferentes tratamentos propostos. Os componentes principais são formados através das relações entre as variáveis, e a rotação "varimax" tem como objetivo concentrar no primeiro fator as variáveis que tem relação entre si e que explicam a maior parte da variança. $\mathrm{O}$ valor de corte para a extração do componente em cada fator foi de 0,50 .

Através da análise fatorial dos componentes principais nos exercícios realizados fora d'água (Tabela 6), observou-se que $82,96 \%$ da variança total é explicada pelos dois fatores (fisiológicos e biomecânicos) em conjunto: que o primeiro fator explica $43,71 \%$ da variança total e o segundo fator explica $39,25 \%$.

Tabela 6 - Explicação total da variança dos exercícios realizados fora d'água.

\begin{tabular}{|c|c|c|c|c|c|c|c|c|c|}
\hline & \multicolumn{3}{|c|}{ Valores Próprios } & \multicolumn{3}{|c|}{$\begin{array}{c}\text { Extração da soma dos } \\
\text { quadrados }\end{array}$} & \multicolumn{3}{|c|}{$\begin{array}{c}\text { Rotação da soma dos } \\
\text { quadrados }\end{array}$} \\
\hline Fator & Total & $\begin{array}{c}\% \mathrm{da} \\
\text { variança }\end{array}$ & $\begin{array}{c}\% \\
\text { acumulado }\end{array}$ & Total & $\begin{array}{c}\% \text { da } \\
\text { variança }\end{array}$ & $\begin{array}{c}\% \\
\text { acumulado }\end{array}$ & Total & $\begin{array}{c}\% \mathrm{da} \\
\text { variança }\end{array}$ & $\begin{array}{c}\% \\
\text { acumulado }\end{array}$ \\
\hline 1 & 3,044 & 50,727 & 50,727 & 3,044 & 50,727 & 50,727 & 2,623 & 43,712 & 43,712 \\
\hline 2 & 1,934 & 32,230 & 82,956 & 1,934 & 32,230 & 82,956 & 2,355 & 39,245 & 82,956 \\
\hline 3 &, 420 & 6,992 & 89,948 & & & & & & \\
\hline 4 &, 350 & 5,828 & 95,776 & & & & & & \\
\hline 5 &, 191 & 3,176 & 98,952 & & & & & & \\
\hline & $\begin{array}{r}6,285 \mathrm{E}- \\
02\end{array}$ & 1,048 & 100,000 & & & & & & \\
\hline
\end{tabular}

Método de Extração: Analises dos componentes principais.

${ }^{a}$ condição experimental $=$ exerc . fora d'água

$\mathrm{O}$ LacF, a FCF e o $\mathrm{VO}_{2} \mathrm{~F}$ tem uma relação alta entre si, o que permitiu agrupá-los em um único fator (fator 1): este fator foi denominado de variáveis fisiológicas (Tabela 7). A FzF e o ImpF também demonstraram uma relação alta entre si, o que permitiu agrupá-los no fator 2 , tendo sido denominado de variáveis biomecânicas. Nos exercícios realizados fora d'água as variáveis fisiológicas tem um maior poder explicativo do fenômeno analisado.

Tabela 7 - Matriz de rotação dos fatores, para as variáveis fisiológicas e biomecânicas dos exercícios realizados fora d'água.

\begin{tabular}{lccc} 
& & Fatores & \\
\hline & 1 & & \\
\hline $\mathrm{VO}_{2} \mathrm{~F}$ &, 897 & \\
$\mathrm{FC}$ &, 886 & \\
$\mathrm{LacF}$ &, 841 & \\
$\mathrm{FzF}$ & &, 934 \\
$\mathrm{ImpF}$ & &, 835 \\
\hline
\end{tabular}

Método de Extração; Analise dos Componentes Principais.

Método de Rotação: Varimax com Normalização Kaiser

a A rotação converge em 3 interações

${ }^{\text {b }}$ condição experimental $=$ exerc. fora d'água

Revista Kinesis, Santa Maria, No Especial, p. 120-154, nov. 2001 
Através da análise fatorial dos componentes principais nos exercícios realizados na profundidade de cicatriz umbilical (Tabela 8), observou-se que $57,54 \%$ da variança total é explicada pelos dois fatores (biomecânicos e fisiológicos) em conjunto: o primeiro fator explica $36,48 \%$ da variança total e o segundo fator explica somente $21,06 \%$.

Na profundidade de cicatriz umbilical, o FzU e o ImpU tem uma relação alta entre si, tendo sido agrupados em um único fator (fator 1), denominado de variáveis biomecânicas (Tabela 9). A FCU e o $\mathrm{VO}_{2} \mathrm{U}$ também demonstraram uma relação alta entre si, sendo agrupados no fator 2, denominado de variáveis fisiológicas. Observou-se entretanto, que a variável LacU não demonstrou uma relação alta com as demais variáveis fisiológicas nesta profundidade de água.

Tabela 8 - Explicação total da variança nos exercícios realizados na profundidade de cicatriz umbilical.

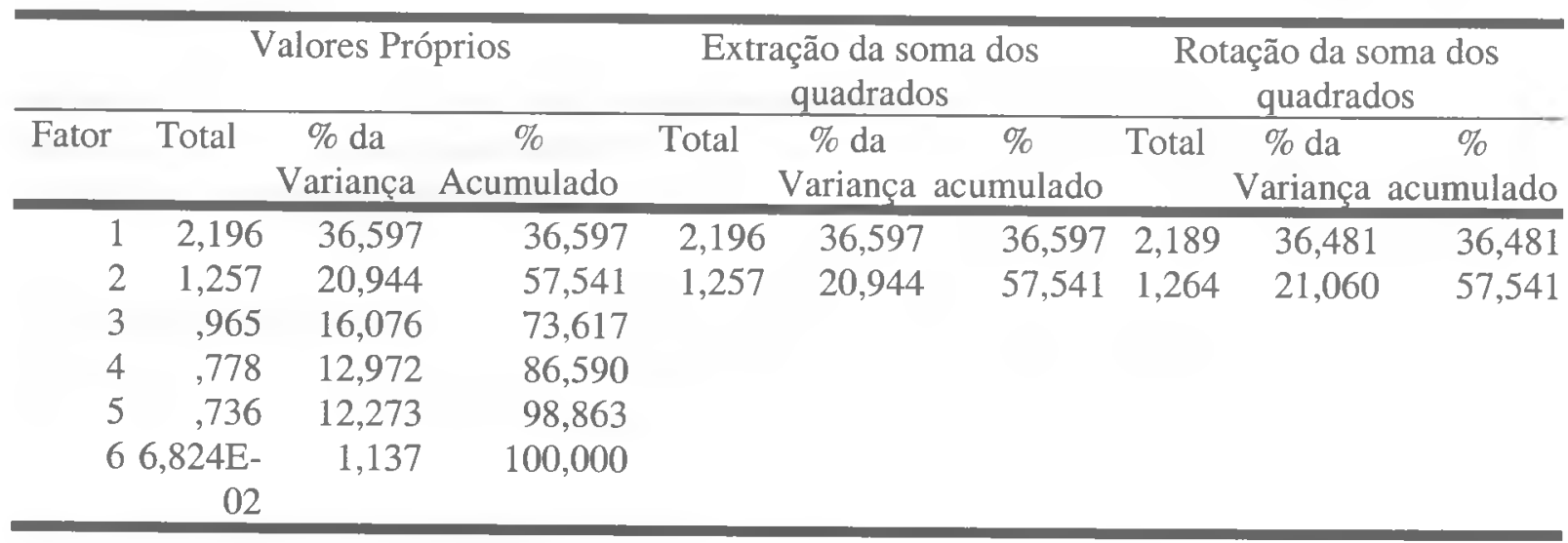

Método de Extração: Analises dos componentes principais.

${ }^{a}$ condição experimental $=$ ex. imerso na profundidade de cicatriz umbilical.

Tabela 9 - Matriz de rotação dos fatores, para as variáveis fisiológicas e biomecânicas dos exercícios realizados na profundidade de cicatriz umbilical.

\begin{tabular}{|c|c|c|c|}
\hline & \multicolumn{3}{|c|}{ Fatores } \\
\hline & 1 & & 2 \\
\hline $\mathrm{FzU}$ & 940 & & \\
\hline $\operatorname{ImpU}$ & 596 & & $y$ \\
\hline LacU & & & $=$ \\
\hline FCU & & $\infty$ & ,759 \\
\hline $\mathrm{VO}_{2} \mathrm{U}$ & & 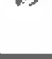 & ,706 \\
\hline
\end{tabular}

Método de Extração; Analise dos Componentes Principais.

Método de Rotação: Varimax com Normalização Kaiser

${ }^{a}$ A rotação converge em 3 interações

${ }^{\mathrm{b}}$ condição experimental $=$ exerc . na profundidade de cicatriz umbilical

Nos exercícios realizados na profundidade de cicatriz umbilical as variáveis biomecânícas tem um maior poder explicativo do fenômeno analisado. 
Tabela 10 - Explicação total da variança nos exercícios realizados na profundidade de ombro.

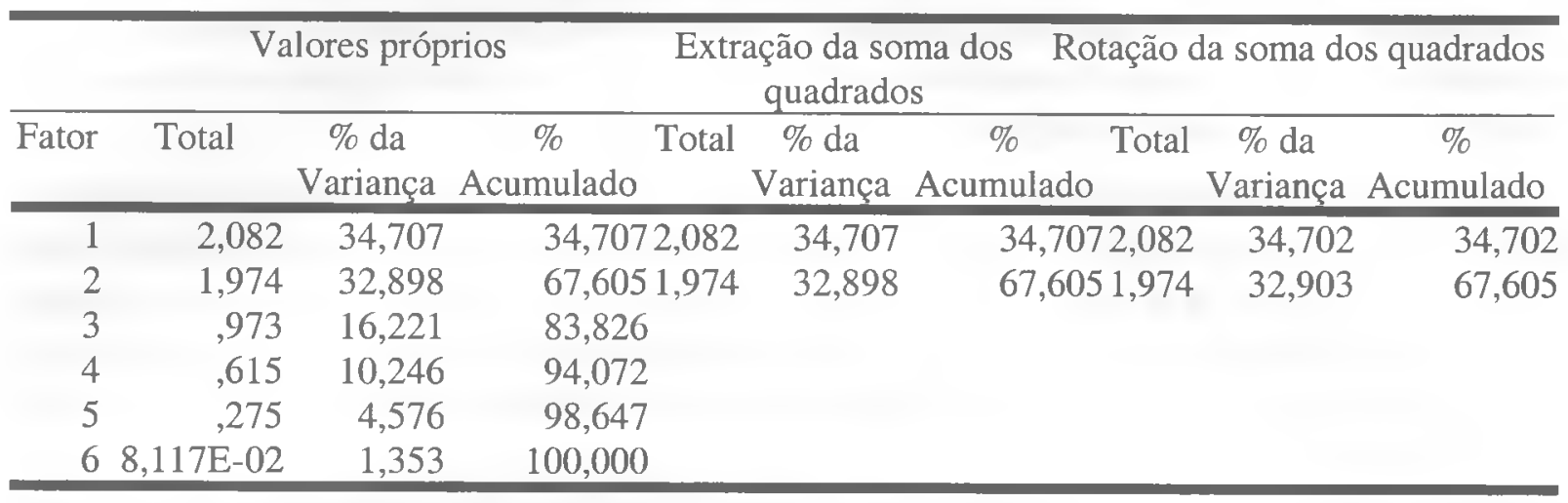

Método de Extração: Analises dos componentes principais.

${ }^{a}$ condição experimental $=$ ex. imerso na profundidade de ombro.

Através da análise fatorial dos componentes principais nos exercícios realizados na profundidade de ombro (Tabela 10), observou-se que $67,61 \%$ da variança total é explicada pelos dois fatores (biomecânicos e fisiológicos) em conjunto. O primeiro fator explica $34,70 \%$ da variança total e o segundo fator explica $32,90 \%$.

No caso da profundidade de ombro, a FzO e o ImpO não demonstraram uma relação alta entre si, tendo sido agrupados em um único fator (fator 1), o qual denominamos de variável biomecânica (Tabela 11). Observou-se entretanto que a variável ImpO demonstrou uma alta relação com as variáveis fisiológicas.

Tabela 11 - Matriz de rotação dos fatores, para as variáveis fisiológicas e biomecânicas dos exercícios realizados na profundidade de ombro.

\begin{tabular}{|c|c|c|}
\hline & \multicolumn{2}{|c|}{ Fatores } \\
\hline & 1 & 2 \\
\hline $\mathrm{FzO}$ & ,945 & \\
\hline $\mathrm{VO}_{2} \mathrm{O}$ & & ,750 \\
\hline $\mathrm{LacO}$ & & ,727 \\
\hline $\mathrm{FCO}$ & & ,721 \\
\hline ImpO & &, 596 \\
\hline
\end{tabular}

Método de Extração; Analise dos Componentes Principais.

Método de Rotação: Varimax com Normalização Kaiser

a A rotação converge em 3 interações

${ }^{b}$ condição experimental $=$ exerc . na profundidade de ombro

$\mathrm{O} \mathrm{VO}_{2} \mathrm{O}$, o LacO e a FCO também demonstraram uma relação alta entre si, o que permitiu agrupá-los no fator 2, denominado de variáveis fisiológicas.

Para verificarmos o grau de relacionamento entre as variáveis utilizamos a correlação linear do Produto Momento de Pearson. A correlação deve responder a 3 perguntas: a) a magnitude e o grau de associação entre as variáveis (r), b) o sentido da correlação e c) se a correlação é significante ou não.

Revista Kinesis, Santa Maria, $N^{\circ}$ Especial, p. 122-154, nov. 2001 
Nas Tabelas 12, 13 e 14, apresenta-se os coeficientes de correlação e o nível de significância da mesma para as variáveis fisiológicas e biomecânicas analisadas nos tratamentos fora d'água, na profundidade de cicatriz umbilical e ombro, respectivamente.

Tabela 12 - Coeficientes de correlação e nível de significância para as variáveis FCF, LacF, $\mathrm{VO}_{2} \mathrm{~F}, \mathrm{ImpF}$ e FzF

\begin{tabular}{rrrrrrr}
\hline & & $\mathrm{FCF}$ & $\mathrm{ImpF}$ & $\mathrm{FzF}$ & $\mathrm{LacF}$ & $\mathrm{VO}_{2} \mathrm{~F}$ \\
\hline $\mathrm{FCF}$ & Correlação de Pearson & 1,000 &,- 180 &, 172 &, $670^{* *}$ &, $721^{* *}$ \\
& Sig. (2-tailed) &, &, 412 &, 432 &, 000 &, 000 \\
$\mathrm{ImpF}$ & Correlação de Pearson &,- 180 & 1,000 &, $616^{* *}$ &,- 082 &,- 210 \\
& Sig. (2-tailed) &, 412 &, &, 002 &, 709 &, 336 \\
$\mathrm{FzF}$ & Correlação de Pearson &, 172 &, $616^{* *}$ & 1,000 &, 350 &, 316 \\
& Sig. (2-tailed) &, 432 &, 002 & &, 101 &, 142 \\
$\mathrm{LacF}$ & Correlação de Pearson &, $670^{* *}$ &,- 082 &, 350 & 1,000 &, $641^{* *}$ \\
& Sig. (2-tailed) &, 000 &, 709 &, 101 & &, 001 \\
$\mathrm{VO}_{2} \mathrm{~F}$ & Correlação de Pearson &, $721^{* *}$ &,- 210 &, 316 &, $641^{* *}$ & 1,000 \\
& Sig. (2-tailed) &, 000 &, 336 &, 142 &, 001 & \\
& & &
\end{tabular}

** Correlação é significante $\mathrm{p}<0.01$ (2-tailed).

${ }^{a}$ condição experimental $=$ exerc. fora d'água

Nos exercícios realizados fora d'água, observaram-se as seguintes correlações estatisticamente significativas: entre a FCF e o LacF; entre a $\mathrm{FCF}$ e o $\mathrm{VO}_{2} \mathrm{~F}$; entre o ImpF e a FzF; e entre o $\mathrm{LacF}$ e o $\mathrm{VO}_{2} \mathrm{~F}$, demonstrando que existe um alto grau de relacionamento entre as três variáveis fisiológicas e também entre as 2 variáveis biomecânicas

Nos exercícios realizados na profundidade de cicatriz umbilical (Tabela 13), observouse que as correlações são diferentes das ocorridas fora d'água: ocorreram somente as correlações estatisticamente significativas entre o ImpU e a FzU, demonstrando que neste tratamento não existe um grau de relacionamento significativo entre as variáveis fisiológicas, e somente um relacionamento significativo entre as variáveis biomecânicas.

Tabela 13 - Coeficientes de correlação e nível de significância para as variáveis FCU, LacU, $\mathrm{VO}_{2} \mathrm{U}, \mathrm{ImpU}$ e $\mathrm{FzU}$

\begin{tabular}{|c|c|c|c|c|c|c|}
\hline & & FCU & ImpU & $\mathrm{FzU}$ & LacU & $\mathrm{VO}_{2} \mathrm{U}$ \\
\hline \multirow[t]{2}{*}{$\mathrm{FCU}$} & Correlação de Pearson & 1,000 & ,059 &, $03 \zeta$ &, 116 &, 190 \\
\hline & Sig. (2-tailed) & & ,791 &, 867 & 609 & ,386 \\
\hline \multirow[t]{2}{*}{$\operatorname{ImpU}$} & Correlação de Pearson & ,059 & 1,000 &, $41^{64}$ &,- 107 &, 000 \\
\hline & Sig. (2-tailed) & ,791 & &, 048 & 637 & 1,000 \\
\hline \multirow[t]{2}{*}{$\mathrm{FzU}$} & Correlação de Pearson &, 037 & 417 & 1,000 &,- 192 &,- 127 \\
\hline & Sig. (2-tailed) & 867 &, 048 & & 391 &, 565 \\
\hline \multirow[t]{2}{*}{$\mathrm{LacU}$} & Correlação de Pearson & 116 &,- 107 &,- 192 & 1,000 &, 017 \\
\hline & Sig. (2-tailed) & 609 & 637 & 391 & &, 942 \\
\hline \multirow[t]{2}{*}{$\mathrm{VO}_{2} \mathrm{U}$} & Correlação de Pearson &, 190 &, 000 &,- 127 & 017 & 1,000 \\
\hline & Sig. (2-tailed) & ,386 & 1,000 &, 565 & ,942 & \\
\hline
\end{tabular}

* Correlação é significante p<0.05 (2-tailed).

${ }^{a}$ condição experimental $=$ ex. imerso no umb 
Nos exercícios realizados na profundidade de ombro (Tabela 14), observou-se que as correlações são diferentes das ocorridas fora d'água e na profundidade de cicatriz umbilical: ocorreram somente as correlações estatisticamente significativas entre $\mathrm{VO}_{2} \mathrm{O}$ e $\mathrm{LacO}$, demonstrando que nesta profundidade não existe um grau de relacionamento significante entre as variáveis fisiológicas, com exceção de $\mathrm{VO}_{2} \mathrm{O}$ e LacO, e que não existe relacionamento entre as variáveis biomecânicas.

Tabela 14 - Coeficientes de correlação e nível de significância para as variáveis FCO, LacO, $\mathrm{VO}_{2} \mathrm{O}, \mathrm{ImpO}$ e $\mathrm{FzO}$

\begin{tabular}{|c|c|c|c|c|c|c|}
\hline & & $\mathrm{FCO}$ & ImpO & $\mathrm{FzO}$ & $\mathrm{LacO}$ & $\mathrm{VO}_{2} \mathrm{O}$ \\
\hline \multirow[t]{2}{*}{ FCO } & Correlação de Pearson & 1,000 &, 360 &, 127 & ,273 &, 365 \\
\hline & Sig. (2-tailed) & &, 091 & ,565 & ,208 & 087 \\
\hline \multirow[t]{2}{*}{ ImpO } & Correlação de Pearson & ,360 & 1,000 &, 154 & ,304 & , 128 \\
\hline & Sig. (2-tailed) & 091 & & ,484 & ,158 &, 560 \\
\hline \multirow[t]{2}{*}{$\mathrm{FzO}$} & Correlação de Pearson & , 127 &, 154 & 1,000 &,- 358 &, 115 \\
\hline & Sig. (2-tailed) &, 565 & 484 & 2900 &, 093 & 601 \\
\hline \multirow[t]{2}{*}{$\mathrm{LacO}$} & Correlação de Pearson & 273 & 304 &,- 358 & 1,000 & $499 *$ \\
\hline & Sig. (2-tailed) & ,208 &, 158 & ,093 & &, 015 \\
\hline \multirow[t]{2}{*}{$\mathrm{VO}_{2} \mathrm{O}$} & Correlação de Pearson & ,365 & , 128 &, 115 &, $499 * *$ & 1,000 \\
\hline & Sig. (2-tailed) & 087 &, 560 & 601 &, 015 & \\
\hline
\end{tabular}

* Correlação é significante $\mathrm{p}<0.05$ (2-tailed).

** Correlação é significante $\mathrm{p}<0.01$ (2-tailed).

${ }^{a}$ condição experimental $=$ ex. imerso no omb

Ao observarmos os resultados das correlações bi-variadas identificamos a necessidade de elaboração de um modelo de análise de regressão para verificarmos o comportamento de algumas variáveis (facilmente observadas e coletadas no dia-a-dia dos profissionais de hidroginástica), e que pudesse explicar o comportamento de uma determinada variável (influenciadora) sobre outras variáveis (influenciadas).

Para analisarmos o grau de dependência entre as variáveis utilizamos a análise de regressão linear, adotando como método de retirada das variáveis influenciadoras, o "stepwise". A regressão nos indica 3 respostas: a) qual a magnitude de influência da variável influenciadora sobre a variável influenciada, b) qual o sinal da influência, se positiva ou negativa e c) se a regressão é significante ou não.

A análise dos modelos propostos pelo "stepwise", da magnitude de influência da variável influenciadora sobre a variável influenciada, consideradas somente aquelas variáveis facilmente coletadas no dia-a-dia dos professores de hidroginástica, não levou a nenhum modelo matemático que satisfizesse as condições propostas.

\section{Conclusão e sugestões}

a) quanto as alterações físiológicas e biomecânicas em diferentes exercícios de hidroginástica:

Revista Kinesis, Santa Maria, $N^{o}$ Especial, p. 124-154, nov. 2001 
- não foram encontradas diferenças estatisticamente significantes entre as variáveis fisiológicas e biomecânicas na realização dos exercícios propostos, mesmo que em cadências de execução diferentes;

a) quanto aos efeitos dos exercícios realizados nas profundidades de água de ombro e de cicatriz umbilical e fora d'água sobre as variáveis fisiológicas:

- o comportamento da freqüência cardíaca e do consumo de oxigênio na realização de exercícios na profundidade de ombro, demonstrou diferenças estatisticamente significantes entre os exercícios executados na profundidade de cicatriz umbilical e também em relação aos exercícios executados fora d'água. Entretanto não foram encontradas diferenças estatisticamente significantes nas variáveis de freqüência cardíaca e consumo de oxigênio entre exercícios realizados fora d'água e exercícios realizados na profundidade de cicatriz umbilical;

- o comportamento das concentrações de lactato sangüíneo demonstrou diferenças estatisticamente significantes entre os exercícios executados dentro e fora d'água, não demonstrando diferenças estatisticamente significantes nas concentrações de lactato entre as diferentes profundidades de água;

a) quanto aos efeitos dos exercícios realizados nas profundidades de água de ombro e de cicatriz umbilical e fora d'água sobre as variáveis biomecânicas:

- As variáveis de força de reação vertical e impulso apresentaram um comportamento semelhante, não tendo sido encontradas diferenças estatisticamente significantes entre os tratamentos realizados nas diferentes profundidades de água, mas demonstrando uma diferença estatisticamente significante entre os tratamentos realizados fora d'água quando comparados com os tratamentos realizados dentro d'água;

O presente estudo vem demonstrar que os indivíduos, quando são submetidos à imersão no meio líquido, apresentam alterações fisiológicas e biomecânicas significantes, tanto em relação aos exercícios executados dentro e fora d'água, como em diferentes profundidades de água.

Os resultados encontrados a partir da análise fatorial dos componentes principais, demonstram que nos exercícios realizados fora d'água as variáveis fisiológicas tem um maior poder explicativo do fenômeno analisado, enquanto que nas profundidades de cicatriz umbilical e ombro são as variáveis biomecânicas que tem um maior poder explicativo dos fenômenos analisados.

Os resultados permitem também afirmar que quanto mais imerso estiver o indivíduo maior será a redução de sua frequiência cardíaca; como consequiêucia o indivíduo conseguirá realizar os exercícios em um menor percentual da frefuiência cardíaca máxima, o que provavelmente será benéfico para determinados tipos de problemas cardíacos.

Os altos índices de consumo de oxigênio, freqüência cardíaca e concentrações de lactato sangüíneo encontrados fora d'água, demonstram uma tendência de uma maior atividade da rota metabólica anaeróbica, enquanto que, quando os exercícios são realizados na profundidade de ombro, mesmo tendo a mesma cadência de execução, demonstram um maior percentual de utilização das rotas aeróbicas.

Os resultados das variáveis biomecânicas encontrados neste estudo, nos permitem afirmar que, quando prescrevermos exercícios com objetivos de proteger e preservar as articulações, os mesmos podem e devem ser realizados dentro do meio líquido, pois os exercícios realizados 
neste meio são classificados de baixo impacto, enquanto que os mesmos exercícios realizados fora d'água são considerados de alto impacto.

Em função dos resultados encontrados, dos conhecimentos adquiridos, bem como das limitações do estudo, sugere-se futuras linhas de pesquisas;

- desenvolver estudos semelhantes em amostras com outras faixas etárias;

- desenvolver estudos semelhantes com outras cadências de exercícios; e

- desenvolver estudos semelhantes em outras profundidades de água.

\section{Referências bibliográficas}

AGOSTINI, E., \& RAHN, H., Abdominal and thoracic pressures at different lung volumes. J. Appl. Physiol. 15:1087-1092, 1960.

AGOSTINI, E.; GURTNWE, G.; TORRI, G., \& RAHN, H., Respiratory mechanics during submersion and negative-pressure breathing. J. Appl. Physiol. 21:251-258, 1996.

AMADIO, A., C., A ciência do esporte: aspectos da biomecânica. Revista Espaço. 1(2):5-9, 1993.

ARBORELIUS, M.; BALDLIN, U., I.; LILJA, B., \& LUNDGREN, C., E., G., Hemodynamic changes in man during immersion with the head above water. Aerospace Med. 43:590$598,1972$.

ARBORELIUS, M.; BALLDIN, U., I.; LILJA, B., \& LUNDGREN, C., E., G., Regional lung function in man during immersion with the head above water. Aerospace Med. 43:701$707,1972$.

AVELLINI, B., A.; SHAPIRO, Y., \& PANDOLF, K., B., Cardio-respiratory physical training in water and on land. Eur J Applied Physiol. 50: 255-263, 1983.

BLOMQVIST, C., G.; NIXON, J., V.; JOHNSON \& MITCHELL, J., H., Early cardiovascular adaptation to zero gravity, simulated by head-down tilt. Acta Astronautica. 7:543-553, 1980.

BLOMQVIST, C., G.; \& STONE, H., L., Cardiovascular adjustments gravitational stress. In: Handbook of Physiology. The Cardiovascular System. Peripheral Circulation and Organ Blood Flow. Gethesda, Md: Am. Physiol. Soc. Vol. III, part 2 chapt. 28, p.1025-1063, 1983.

BULLARD, R., W., \& RAPP, G., M., Problems of body heart loss in water immersion. Aerospace Med. 41:1269-1277, 1970.

CASSADY, S., L., \& NIELSEN, D., H., Cardiorespiratory respohses of healthy subjects to calisthenics performed on land versus in water. Physical Therapy, 72(7):532-538, 1992.

CHOUKROUN, M., L., \& VARENE, P., Adjustments in oxygen transport during head-out immersion in water at different temperatures. J. Appl. Physiol. 68(4):1475-1480, 1990.

CORSINO, E., L.; LÓPES, R., S., \& PÉRES, M., A., R., La Evaluación de la Respuesta Fisiológica y Metabólica Mediante Procedimentos Ergométricos: Ambiente Aire vs Ambiente Agua. Archivos de Medicina Del Deporte. Vol. XII. 45:29-38, 1995.

COSTILL, D., L.; CAHILL, P., J., \& EDDY, D., Metabolic responses to submaximal exercise in three water temperatures. J. Appl. Physiol. 22:628-632, 1967.

COSTILL, D., L., Energy requirements during exercise in the water. J Sports Med. Phys. Fitness. 11:87-91, 1971 .

Revista Kinesis, Santa Maria, $N^{o}$ Especial, p. 126-154, nov. 2001 
CUNHA, S., A.; BRENZIKOFER, R., \& LIMA FILHO, E., C., Metodologia para análise biomecânica dos fundamentos do futebol. IN.: Anais do VI Congresso Brasileiro de Biomecânica. Brasilia. Pp.:27-33, 1995.

DENISON, D., M.; WAGNER, P., D.; KINGABY, G., L., \& WEST, J., B., Cardiorespiratory responses to exercise in air and underwater. J. Appl. Physiol. 33(4):426-430, 1972.

ECHT, M.; LANGE, L., \& GAUER, O., H., Changes of peripheral venous tone and central transmural venous pressure during immersion in a thermo- neutral bath. Pfügers Arch. 352:211-217, 1974.

EVANS, B., W.; CURETON, K., J., \& PURVIS, J., W., Metabolic and circulatory responses to walking and jogging in water. Research Quarterly. 49:442-449, 1978.

FOLINSBEE, L., Cardiovascular response to apneic immersion in cool and warm water. J. Appl. Physiol. 36:226-232, 1974.

FRANCIS, P., R.; LEIGH, M., \& BERZINS, A, Shock absorbing characteristics of floors used for dance exercise. International Journal of Sport Biomechanics. 4:282-305, 1988.

GAUER, O., H., \& HENRY, J., P., Neurohormonal control of plasma volume. Intern. Rev. Physiol. 9:145-190, 1976.

GLEIM, G., W., \& NICHOLAS, J., A., Metabolic costs and heart rate responses to treadmill walking in water at different depths and temperatures. The American Journal of Sports Medicine. 17(2):248-252, 1989.

GREENLEAF, J., E.; DUNN, E., R.; NESVIG, C.; KEIL, L., C.; HARRISON, M., H.; GEELEN, G., \& KRAVIL, S., E., Effect of longitudinal physical training and water immersion on orthostatic tolerance in men. Avit. Space Environ Med. 59:152-159, 1988.

HAMER, P., W., \& MORTON, A., R., Water-Running: Training Effects and Specificity of Aerobic-Anaerobic and Muscular Parameters Following an Eight-Week Interval Training Programme. Australian J. of Science and Med. in Sport. 22(1):13-22, 1990.

HEIGENHAUSER, G., F.; BOULET, D.; MILLER, B., \& FAULKNER, J., A., Cardiac outputs of post-myocardial infarction patients during swimming and cycling. Med. Sci. in Sports Exercise. 9(3):143-147, 1977.

HÖLMER, L., \& BERGH, V., Metabolic and thermal response to swimming in water at varying temperatures. J. Appl. Physiol. 37:702-705, 1974.

KAWAKAMI, Y.; NATELSON, B., H., \& DuBORS, A., B., Cardiovascular effects of face immersion and factors affeting diving reflex in man. J. Appl. Physiol. 23:964-970, 1967.

KEATINGE, W., R., \& EVANS, M., The respiratory and cardiovascular response to immersion in cold and warm water. Quart. J. Exptl Physiol. 46:83-94, 1961.

KRUEL, L., F., K., (1994). Peso Hidrostático e Freqüiência Cardíaca em Pessoas Submetidas a Diferentes Profundidades de Água. Dissertação de Mestrado. Universidade Federal de Santa Maria, 1994

KRUEL, L., F., M.; TAMAGNA, A., \& QUINTAS, J., P., R., Determinação de forças produzidas por pessoas realizando exercícios aeróbicos. IN.: Anais do VI Congresso Brasileiro de Biomecânica. Brasília. Pp.:119-124, 1995.

KRUEL, L., F., M.; DIAS, A., B., C.; SILVA, R., C.; TARTARUGA, L., A., P.; PICANÇO, P., S., \& RANGEL, A., B., Determinação da Freqüência Cardíaca em pessoas de diferentes 
idades submetidas a diferentes profundidades de água. IN.: Anais do Simpósio Internacional de Ciência e Tecnologia no Esporte. Poa. Multimedia. Ed. Grafica, 1997.

LANGE, L.; LANGE, S.; ECHT, M., \& GAUER, O., H., Heart volume in relation to body posture and immersion in a thermo-neutral bath. Pfluegers Arch. 353:219-226, 1974.

LAZZARI, J., M., A., \& MEYER, F., Freqüência cardíaca e percepção de esforço na caminhada aquática e na esteira em mulheres sedentárias e com diferentes percentuais de gordura. Revista Brasileira de Atividade Física e Saúde. 2(3):07-13, 1997.

LOPES, E. W., SILVEIRA, L., R.; FARIA, O., B.; GOBATTO, C., A., \& CUNHA, S., A., Utilização de variáveis cinemáticas correlacionadas a parâmetros metabólicos no futebol. In.: Anais do VI Congresso Brasileiro de Biomecânica. Maio, Pp.:20-26, 1995.

McARDLE, W., D.; MAGEL, J., R.; LESMES, G., R., \& PECHAR, G., S., Metabolic and cardiovascular adjustment to work in air and water at 18, 25 and $33^{\circ} \mathrm{C}$. J. Appl. Physiol. 40:85-90, 1976.

McMURRAY, R., G., \& HORVATH, S., M., Thermoregulation in swimmers and runners. J. Appl. Physiol. 46:1086-1092, 1979.

MICHAUD, T., H.; RODRIGUES-ZAYAS, J.; ARMSTRONG, C., \& HARTNIG, M., Ground reaction forces in high and low impact aerobic dance. The Journal of Sports Medicine and Physical Fitness. 33:359-366, 1993.

MORAES, E., Z., C., Metodologia de medida de esforço para exercícios de hidroginástica em diferentes profundidades de água. Monografia de Especialização. Universidade Federal de Santa Maria, 1998.

OLIVEIRA, J., A.; AMADIO, A., C., \& MANOEL, E., J., Estudo cinemático na interpretação coordenativa da habilidade de arremessar. IN.: Anais do VI Congresso Brasileiro de Biomecânica. Brasília. Pp.:71-77, 1995.

OZGUVEN, H., N., \& BERNE, N., Na experimental and analytical study of impact forcer during human junping. Journal of Biomechanics. 21:1061-1066, 1998.

RENNIE, D., W.; DiPRANPERO, P., \& CERRETELLI, P., Effects of water immersion o cardiac output, heart rate and stroke volume of men at rest and during exercise. Med. Sport (Turin). 24:223-228, 1971.

ROESLER, H., Desenvolvimento de plataforma subaquática para medições de forças e momentos nos três eixos coordenados para utilização em biomecânica. Tese de Doutorado. Programa de Pós-Graduação em Engenhąria Mecânica. UFRGS, 1997.

SACCO, I., C., N., \& AMADIO, A., C., Proposta metodológicał łara o estudo de respostas biomecânicas aplicadas na avaliação do andar em indi yíduos portadores de diabetes. In.: Anais do VI Congresso Brasileiro de Biomecânica. Brasília. Pp.:78-84, 1995.

SHELDAHL, L., M.; WANN, L., S.; CLIFFORD, O., S.; TRISTANI, F., E.; WOLF, L., G., \& KALBELEISH, J., H., Effect of central hipervolemia on cardiac performance during exercise. J. Appl. Physiol.: Respirat. Environ. Exerc. Physiol. 52:1662-1667, 1984.

SHELDAH, L., M., Special ergometric techniques and weight reduction. Med. Sci. Sports Exerc. 18:25-30, 1985.

TOWN, G., P., \& BRADLEY, S., S., Maximal metabolic responses of deep and shallow water running in trained runners. Medicine and Science in Sports and Exercise. 23(2):238-241, 1991.

VALIANT, G., A., \& CAVANAGH, P., R., A study of landing from jump: Implications for the

Revista Kinesis, Santa Maria, $N^{o}$ Especial, p. 128-154, nov. 2001 
design of a basketball shoe. In.: Winter, D. A. \& Norman, R. W. (Eds.), Biomechanics IXB, Pp.:177-122, Champaign, Human Kinetics, 1985.

VICKERY, S., R.; CURETON, K., J., \& LANGSTAFF, J., L., Heart rate and energy expenditure during aqua dynamics. The Physician and Sportsmedicine. 11:62-72, 1983.

WHITLEY, J., D., \& SCHOENE, L., L., Comparison of heart rate responses - water walking versus treadmill walking. Journal of The American Physical Therapy Association. 67(I0):1501-1504, 1987.

WILBER, R., L.; MOFFATT, R., J.; SCOTT, B., E.; LEE, D., T., \& CUCUZZO, N., A., Influence of water run training on the maintenance of aerobic performance. Med. Sci. Sports Exerc. 28(8):1056-1062, 1996.

WILDER, R., P.; \& BRENNAN, D., K., Physiological responses to deep water running in athletes. Sports Med. 16:374-380, 1993.

WILDER, R., D.; BRENNAN, D., K., \& SCHOTTE, D., E., A standard measure for exercise prescription for aqua running. Am. J. Sports Med. 21:45-48, 1993.

ZATSIORSKY, V., M., \& FORTNEY, V., L., Sport Biomechanics 2000. Journal of Sports Sciences. 11(4):279-283, 1993.

\title{
Physiological and biomechanical alterations in individuals practicing water exercises inside and outside of the water
}

\begin{abstract}
The purpose of this work was to compare physiological and biomechanical alterations during water exercises performed outside of the water (FD) and at navel scar (PCU) and shoulders (PO) deep level in the water. Sample was composed of 23 female subjects averaging $54 \pm 11,16$ years of age, practitioners of water gymnastics in the Physical Education each kind of water exercity of Rio Grande do Sul. In the beginning the sample was divided into 5 groups (one for Heron, Lobster, Alligator I drawn from the most used ones by the Brazilian professors). The exercises selected were was performed during 5 minutes being Pelican. Experimental groups formation was done randomly. Each exercise heart rate (FC), buting 5 minutes being the recovery time determined individually. The analyzed variables were: impulse (Imp). Several statistic trentration (Lac), oxygen consumption $\left(\mathrm{VO}_{2}\right)$, veffical reaction strength $(\mathrm{Fz})$ and to localize the differences, to verify thents were used in order to compare theclasses of the discriminating variables, determined the influence leverify the correlation level between physiological and biomechanical variables and to 8.0. There were no rignifiel of one variable upon another, using the SPSS statistical package for windows, version the exception of the outside of the behavior with values being his the did not show significant statistical differe the water and diminishing as the immersion was increasing $\mathrm{VO}_{2}$ and $\mathrm{FC}$ between $\mathrm{PO}$ and the other two treal difference between FD and PCU, but were different when comparison was made FD, the physiological variables had more power to it was for variables Lac, Imp, and FZ. In the exercises performed and $\mathrm{PO}$ ) the biomechanical variables explain it better.
\end{abstract}

Keywords: water aerobics; heart frequency; consumption of oxygen. 